University of Louisville

ThinkIR: The University of Louisville's Institutional Repository

Electronic Theses and Dissertations

$5-2009$

\title{
Oral status of residents of long-term care facilities in Kentucky.
}

Lynn Donnelly Austin

University of Louisville

Follow this and additional works at: https://ir.library.louisville.edu/etd

\section{Recommended Citation}

Austin, Lynn Donnelly, "Oral status of residents of long-term care facilities in Kentucky." (2009). Electronic Theses and Dissertations. Paper 58.

https://doi.org/10.18297/etd/58

This Doctoral Dissertation is brought to you for free and open access by ThinkIR: The University of Louisville's Institutional Repository. It has been accepted for inclusion in Electronic Theses and Dissertations by an authorized administrator of ThinkIR: The University of Louisville's Institutional Repository. This title appears here courtesy of the author, who has retained all other copyrights. For more information, please contact thinkir@louisville.edu. 


\title{
ORAL STATUS OF RESIDENTS OF LONG-TERM CARE FACILITIES IN KENTUCKY
}

\author{
By \\ Lynn Donnelly Austin \\ B.S., University of Michigan, 1978 \\ M.P.H., University of Michigan, 1981

\begin{abstract}
A Dissertation
Submitted to the Faculty of the

Graduate School of the University of Louisville

and

Graduate Studies and Research at Western Kentucky University

In Partial Fulfillment of the Requirements

For the Degree of
\end{abstract} \\ Doctor of Philosophy \\ Department of Leadership, Foundations, and Human Resource Education \\ University of Louisville \\ and \\ College of Education and Behavioral Sciences \\ Western Kentucky University
}

May 2009 


\title{
ORAL STATUS OF RESIDENTS OF LONG-TERM \\ CARE FACILITIES IN KENTUCKY
}

\section{By}

\author{
Lynn D. Austin \\ B.S., University of Michigan, 1978 \\ M.P.H., University of Michigan, 1981
}

A Dissertation Approved on

March 26, 2009

By the following Dissertation Committee:

Dissertation Director 


\section{DEDICATION}

This dissertation is dedicated to my husband, Bob, for continued love and support and to my children, Nick, Jeff, and Greg, for the boundless joy they bring to my life every day. It is also dedicated to my parents, Barbara Donnelly and the late James Donnelly, for providing me with invaluable educational opportunities. 


\section{ACKNOWLEDGEMENTS}

This dissertation would not have been possible without the patient guidance of Dr. Ric

Keaster. His drive and determination in seeing this project through to the end is typical of his professional and personal integrity. The help of my methodologist, Dr. Reagan Brown was also invaluable. Additionally, Dr. Lisa Durham was extremely gracious in allowing me access to data from the 2005 Kentucky Elder Oral Health Survey. 


\section{ABSTRACT \\ ORAL STATUS OF RESIDENTS OF LONG-TERM CARE \\ FACILITIES IN KENTUCKY}

Lynn D. Austin

March 26, 2009

Oral health is a critical component of every individual's general health and wellbeing. There is evidence that consistently demonstrates that age, poverty, education levels, minority status, and living in a rural area are risk factors for poorer oral status. In Kentucky, it is projected that there will continue to be an increase in the rural population and a decrease in the urban population; this projection is more pronounced for the aged 65 and over cohort. In 2005, a state-wide oral health survey (KEOHS) which assessed the oral health status and treatment needs of residents aged 65 and over was completed. The KEOHS gathered information on homebound residents, long-term care facility residents, and independently-living adults. To date, only data pertaining to the homebound residents have been analyzed. The focus of this study, therefore, was on data involving residents of long-term care facilities and assisted living facilities. Specifically, it will be determined if there is a relation between actual oral health and self-reported oral health as well as the nature of the relationship between oral health status and the type of community. 
The study group was comprised of a total of 912 individuals from 27 nursing homes and assisted living facilities around the state. Every elder who participated was given the questionnaire (or interviewed by the research team) and the clinical examination performed by a trained and calibrated dentist.

The first study question concerned the agreement between an individual's selfreported oral health status (excellent, good, fair, or poor) and the examiner evaluation of that same individual's oral health status. Analyses revealed a significant correlation between an individual's self-reported oral status and the examiner-reported oral health status of the same individual.

The second research question concerned the relation between the examinerreported oral health status and place of residence prior to entering a nursing home. Analyses indicated that there was not a significant difference in oral health based upon place of residence prior to entering a nursing home.

Further research is necessary to determine the change in oral health status of the individuals who were examined in 2005. If it were shown that individuals who had higher clinician-rated oral health status at admission maintained their oral health better than individuals with lower clinician-rated oral health status, there would be additional support for development of policy that mandated a minimal level of oral health at admission to a long-term care facility. 


\section{TABLE OF CONTENTS}

PAGE

ACKNOWLEDGEMENTS. . . . . . . . . . . . . . . . . . .

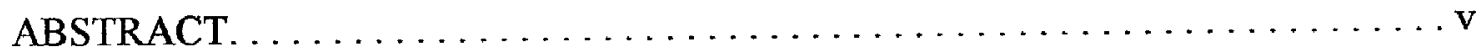

CHAPTER

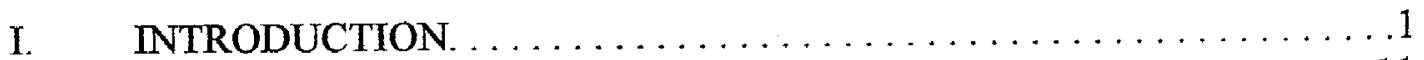

II. REVIEW OF THE LITERATURE $\ldots \ldots \ldots \ldots \ldots \ldots \ldots \ldots \ldots \ldots \ldots$

III. METHODOLOGY . . . . . . . . . . . . . . . . . . . 91

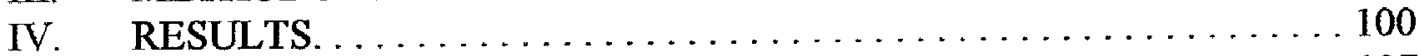

V. DISCUSSION. . . . . . . . . . . . . . . . . . 107

REFERENCES. . . . . . . . . . . . . . . . . . . 118

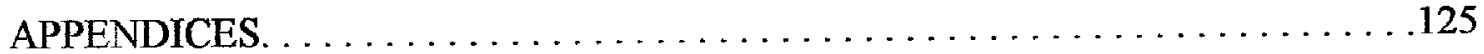

CURRICULUM VITAE. . . . . . . . . . . . . . . . . . 142 


\section{CHAPTER 1}

\section{INTRODUCTION}

Oral health is a critical component of every individual's general health and wellbeing. Oral health, however, means more than just an attractive smile. Poor oral health and untreated oral diseases and conditions can have a significant impact on quality of life. According to the recently released Surgeon General's report on oral health in America, a large percentage of the population suffers from a reduced quality of life due to oral and facial pain. This pain is largely due to infections of the gums that support the teeth and can lead to tooth loss. This finding is especially critical since more than $75 \%$ of the population is affected by some type of periodontal disease or gingivitis (nidcr.nih.gov, 2008).

In addition to oral pain, oral health has also been shown to affect systemic health regarcless of an individual's age. Underscoring the importance of the relation between oral and systemic health are several important findings. One such finding is the link between poor oral health and bacterial endocarditis, a condition in which the lining of the heart and heart valves become inflamed. Paster et al. (2001) found that individuals with poor oral health had a significantly increased likelihood of developing bacterial endocarditis, a debilitating, often fatal disease. Poor mouth care also can contribute to oral cancer, which takes more lives annually than cervical or skin cancer (Franco et al., 1999). Additionally, poor oral health affects the digestive process, which begins with physical and chemical activities in the mouth. Left untreated, digestive tract problems can 
result in intestinal failure and Irritable Bowel Syndrome (healthywomen. org, 2008).

Evidence of the importance of oral health also includes the relation between poor oral health and pre-term birth or low birth weight. Women with poor periodontal health have a 3.5 times greater risk of having a pre-term birth or delivering a baby with low birth weight than women with healthy oral tissue (Lopez, Smith, \& Gutierrez, 2002).

Especially critical for an elderly individual, however, are the findings linking poor oral health to heart disease and diabetes mellitus. Individuals with poor oral health have a $25 \%$ increased risk of coronary heart disease as compared to individuals with optimal oral health (Hujoel, Drangsholt, Spiekerman, \& DeRouen, 2000). Regarding a relation with diabetes mellitus, researchers have found that individuals with poor oral health, especially poor periodontal health, have a 3 times greater risk of developing diabetes than an individual with optimal periodontal health (Grossi \& Genco, 1998).

\section{Significance}

According to U. S. Census Bureau (2008) projections, the 65 and older population will increase from $12.97 \%$ of the total population in 2010 to $20.17 \%$ of the total population in 2050 . Further, it is projected that the 85 and older population will increase from $1.85 \%$ of the total population in 2010 to $4.34 \%$ of the total population in 2050 . Reasons for the shift in population demographics include an increased lifespan and a decline in the birth rate. This shift in the U.S. population has been coupled with an increased understanding of the concept of healthier aging including lifetime oral health. The outdated concept of oral health was premised on the notion that teeth were not meant to last a lifetime. Recent developments including community water fluoridation and the application of pit and fissure sealants have helped to redefine oral health. This redefined 
concept of healthier aging underscores the importance of establishing appropriate oral health programs for the elderly.

In Kentucky, it is projected that there will continue to be an increase in the rural population and a decrease in the urban population; this projection is more pronounced for the aged 65 and over cohort (U. S. Census Bureau, 2008). These projections are of critical importance because rural individuals are less likely than individuals who reside in urban areas to have optimal oral health (Adams, Slack-Smith, Larson, \& O'Grady, 2004). Additionally, individuals who reside in rural areas are less likely to have dental insurance (Vargas, Yellowitz, \& Hayes, 2003).

These shifts in the number of people in the 65 and over cohort, coupled with the increasing number of rural Kentuckians, call attention to the importance of accurate assessments of the oral health of elderly individuals. An overarching consideration is the need to develop appropriate public policy to ensure that this segment of the population receives regular assessments and, when appropriate, necessary interventions. Without appropriate interventions, these individuals are far more likely to have decrements in systemic health resulting in increased need for hospitalizations as well as a shortened lifespan (Isaksson \& Söderfeldt, 2007)

\section{Societal Considerations Prompting Study}

In 2005, the University of Kentucky School of Dentistry, in conjunction with the Office of Oral Health of the Department for Public Health, Commonwealth of Kentucky, completed the Kentucky Elder Oral Health Survey (KEOHS). The KEOHS was a statewide oral health survey which assessed the oral health status and treatment needs of residents aged 65 and over. Because of the 2005 Elder Survey, quite a bit is known about the 
oral health status of the elderly but the questions that this study will address will add to that body of knowledge.

Most data concerning the oral health of Kentucky's elderly population are based on a Centers for Disease Control and Prevention (CDC) study known as the Behavioral Risk Factor Surveillance System (BRFFS, 2008). The BRFSS is a telephone survey which has been conducted by the CDC since 1984. There are, however, several limitations. These limitations include the exclusion of nursing homes and other long-term care facilities from their survey. Additionally, since it is a telephone survey, individuals without phone service were not included in the survey data. This is especially important since rural elderly individuals are less likely to have telephone service than urban elderly individuals (BRFFS, 2008). Last, since nursing home populations were excluded, the findings may not be representative of the entire elderly population. The sample likely contained individuals who were healthier than their institutionalized counterparts.

One of the hypotheses for this dissertation is that individuals from rural areas have poorer oral health than individuals from urban areas. Since Kentucky will continue to realize a shift from urban to rural areas of living, it is imperative that data concerning this population be analyzed. If the hypothesis is shown to be correct, there would be increased impetus to develop policy regarding regular oral assessments of an elderly individual. Additionally, some studies (Berkey \& Berg, 2001; Vargas, Yellowitz, \& Hayes, 2003) espoused the importance of ensuring that schools that educate dental professionals should include an expanded geriatric dentistry curriculum to improve the likelihood of practicing professionals reaching out to elderly individuals. 


\section{Purpose of the Study}

The purpose of this study was twofold. First, the researcher was interested in determining how accurately self-reported accounts of oral health status correlate with the actual oral health status of elders. Data have been collected on these two factors, but have not been systematically analyzed. Without analysis of these data, there is no way to a) tell if these two correlate or b) make recommendations based on any findings regarding the assumptions that are made in these circumstances.

Second, the researcher wished to determine if there was a difference in the oral health of Kentuckians based upon area of residence prior to entering a long-term care facility, i.e., elders from areas defined as rural, urban, and small cities. The literature suggests that differences do exist across these populations and the researcher wanted to see if these data from Kentucky reflect what the current literature suggests.

\section{Research Questions/Hypotheses}

There are two research questions that the researcher addressed in this dissertation. The first concerned the degree of consistency between self-reported accounts of oral health status and the actual oral health status of elders. The null hypothesis related to this research question is that there is no correlation between self-reported accounts and actual oral health status of elders. The second research question concerned whether differences exist in the oral health of Kentuckians based upon area of residence prior to entering a long-term care facility, i.e., elders from areas defined as rural, urban, and small cities. The null hypothesis related to this research question is that there is no difference in oral health status based upon previous area of residence. 


\section{General Methodology}

This study is an analysis of existing data. In 2005, the University of Louisville School of Dentistry, in conjunction with the Office of Oral Health of the Department for Public Health, Commonwealth of Kentucky, completed the Kentucky Elder Oral Health Survey (KEOHS). The specific purposes of the KEOHS were to (a) evaluate the oral health status of elders living in different environmental contexts, (b) assess perceived and actual oral health needs, (c) identify factors affecting utilization of dental services within this population, (d) identify social and personal characteristics that affect oral health status, (e) determine treatment needs of this population, and (f) develop a model to address the oral health needs of this population. The original intended use of these data was to develop a more accurate depiction of the oral health status of well elders in a community. No formal report was ever written, however, analyzing the remaining data including urban or rural residence as a factor in an individual's oral health status.

Approximately one-third of the individuals were from rural areas, one-third of the individuals were from small cities, and one-third of the individuals were from urban areas. A face-to-face survey containing close-ended questions was completed for each individual. The survey consisted of four basic components: demographic information, general health questions, utilization of dental services, and self-reported oral health status. This provided important information concerning the relative importance of dental health to elderly individuals. A clinical examination was then performed to validate the survey and to assess actual oral health status and actual treatment needs. Four groups were included in the 2005 KEOHS study: (a) elders living independently, (b) elders 
living independently who utilize senior centers, (c) homebound elders, and (d) nursing home residents.

As a result of the $2005 \mathrm{KEOHS}$, additional information has been gathered concerning the homebound elder population. Data concerning nursing home residents, however, have not been analyzed. The nursing home residents, therefore, will be the focus of this dissertation. Correlation techniques were used to determine agreement between self-reported oral health status and actual oral health status. Simple regression analyses were used to determine if place of residence prior to entering a long-term care facility was a significant factor in an individual's oral health status. All data were analyzed using SPSS 16.0 .

\section{Definitions}

The following definitions are provided for the purposes of clarity in the remaining chapters of this report. Most are technical terms used in dentistry. All definitions were taken from Stedman's Medical Dictionary, 27th edition (Stedman, 2000).

Activities of Daily Living (ADLs): six basic activities that support survival and are designed to measure functional ability including (a) eating, (b) getting in and out of bed, (c) getting around inside, (d) dressing, (e) bathing, and (f) toileting. A person is considered disabled on an ADL activity if he or she is unable to perform the activity, uses active help, uses equipment, or requires standby help.

Alveolar bone: the thickened ridge of bone that contains the tooth sockets on bones that bear teeth

Calculus: calcified deposits on the teeth, formed by the continuous presence of dental plaque 
Caries: cavities; gradual or acute decay and disintegration of soft or calcified dental tissue

Crown (coronal): the visible portion of the tooth above the gumline Dental plaque: usually colorless biofilm that builds up on the teeth Dentate: having teeth

Dysgeusia: the distortion or decrease of the sense of taste

Dysphagia: difficulty in swallowing

Edentulous: without teeth

Gingiva: the mucosal tissue that lies over the alveolar bone

Gingival pocket: when the depth of the sulcus has moved towards the root of the adjacent tooth, but has not yet breached the connective tissue fibers that connect the gingiva to the tooth

Gingivitis: inflammation of the gums characterized by redness, swelling, and tendency to bleed

Homebound: person with limitations in mobility that restrict the ability to go outside of the home alone and or the inability to perform ADLs by self

Mandibular: pertaining to the lower dental arch

Maxillary: pertaining to the upper dental arch

Mucosa (mucous membranes): are linings of mostly endodermal origin, covered in epithelium, which are involved in absorption and secretion

Periodontal disease: a chronic bacterial infection that affects the gums and bone supporting the teeth 
Periodontitis: involves progressive loss of the alveolar bone around the teeth, and if left untreated, can lead to the loosening and subsequent loss of teeth

Root caries: caries found on the root surfaces of teeth

Streptococcus mutans: gram-positive, anaerobic bacteria commonly found in the human oral cavity and is a significant contributor to tooth decay

Xerostomia: subjective complaint or clinical assessment of dry mouth caused by the impairment of normal salivary flow

\section{Definitions of Areas of Residence}

For the purposes of this study, a modification of the Washington State Department of Health (2001) definitions of areas of residence was used.

Rural Areas: towns with populations below 10,000 and other isolated rural areas

Small cities: towns with populations between 10,000 and 49,999 and surrounding rural areas with high commuting levels to these towns

Urban Core Areas: continuously built up areas of 50,000 persons or more. These areas correspond to the US Bureau of the Census defined Urbanized Areas.

Assumptions

As the researcher in this study, it was assumed that the results of the 2005 study were accurate. The clinical data were collected by Dr. Robert G. Henry of the University of Kentucky. Individuals involved in the collection of questionnaire data were Dr. Robert Henry, Dr. Nancy Sallee, and Lisa Durham. The researchers were diligent in protecting the integrity of the data. 


\section{Limitations}

A limitation of this study includes external validity, or the generalizability, of the study. While it is likely that similar results may be found among elderly individuals in other long-term care facilities and assisted living facilities, the fact that the participants in the 2005 study were a convenience sample may make it difficult to conclusively apply these results to other populations. There may have been reasons why many of the nursing homes and assisted living facilities in Kentucky chose not to participate. Some of these reasons may include concern over potentially-deleterious reports if administrators of these nursing homes believed that the oral status of their residents was below standards of optimal oral health. 


\section{CHAPTER II}

\section{REVIEW OF THE LITERATURE}

A review of the literature concerning oral health status of older individuals involves several critical elements. These elements include the status of institutionalized individuals as well as variables related to oral health. International findings as well as United States findings will be presented with respect to institutionalized individuals. Variables relevant to oral health will also be examined including nutritional status, longevity, and place of residence. This chapter will examine what is currently known in the field as well as describe areas that need further research.

Theoretical Framework

Several theories exist regarding quality of life issues. Unfortunately, research specifically addressing the four topics of interest (older Americans, dental care, organizational structure, and outcomes) is virtually nonexistent. Thus, practical considerations mandate that a broader perspective be taken to review the more general knowledge about the relationship between organizational structure and outcomes of oral health care across provider settings.

The major theory addressing the relationship between structure and outcome is Donabedian's Structure/Process/Outcome Model. From this work, the implications for the provision of primary care to elder Americans including the effects of evolving organizational structures will be considered. The Structure/Process/Outcome Model 
addresses aspects of health care more appropriately and, therefore, will serve as the theoretical framework for this review of the literature.

\section{Structure/Process/Outcome Model}

Avedis Donabedian's Structure/Process/Outcome (SPO) model is cited in research on measures of healthcare quality (Burns, 1995; Donabedian, 1966). Donabedian characterizes structural measures of quality as the professional and organizational resources allied with the provision of care. These measures incorporate credentials of personnel and operating capacities of facilities. Specifically, it is important to understand what resources are available in order to implement measurable change in the oral health status of older adults. In the situation involving elders living in long-term care facilities, appropriate modifications will need to come from outside the structure as resources are quite limited. Modifications in the current structure of oral health care delivery to elderly individuals could involve changes in policy regarding a minimal level of acceptable oral health at admission to a long-term care facility. Process measures of quality refer to the things done to and for the patient by practitioners in the course of treatment (Gustafson \& Hundt, 1995). Ontcome measures are the desired states resulting from care processes, which may include reduction in morbidity and mortality, and improvement in the quality of life (Kane \& Kane, 1988). Current process measures are appropriate to effect changes in the outcome. The existing structure of oral health care delivery to elderly individuals must be emphasized, however, in order to realize improvements in the outcome. The development of appropriate policy from governmental agencies and educational institutions will help to lay the groundwork to ensure meaningful policy changes occur. Donabedian (1988) noted a distinction between technical and interpersonal outcomes. 
Technical outcomes encompass the physical and functional aspects of care. Interpersonal results include aspects of the art of medicine. Within Donabedian's framework, these two types of outcomes are interdependent, so that one cannot be considered separately from the other in evaluating the quality of care.

Donabedian's Structure/Process/Outcome Model serves as an appropriate framework in which to examine quality of life factors as related to the oral health status of elderly individuals. The focus of this review of the literature will be on the status of institutionalized patients, both internationally and in the United States; oral health and related variables; and self-perception of oral status of elderly individuals.

The great majority of the literature reveals that older individuals have poor oral health. If a more appropriate process for meeting treatment needs of the elderly can be realized, oral health outcomes can be vastly improved. This process would include regular assessments of oral health status as well as a thorough oral examination prior to admittance to a long-term care facility. These process improvements would naturally lead to improvements in outcomes: higher quality nutrition which, among other influences, affects quality of life and life span.

\section{Oral Status of Institutionalized Patients}

\section{International Findings}

Andersson, Hallberg, Lorefält, Unosson, and Renvert (2004), investigated the occurrence of oral health problems noted in elderly rehabilitation patients during the admission process. Because systemic assessments seldom include oral assessments, many elderly rehabilitation patients have oral concerns which go unnoticed. The researchers also sought to determine if an association existed among oral health problems and age, 
gender, living conditions, reason for admission, number of prescribed medications, and nutritional status.

The sample for this study consisted of patients $(N=161)$ who had been admitted to one of three wards at a hospital in southern Sweden between November 1996 and January 1998. The investigators, however, did not provide information regarding the total population of patients at these wards. The wards were a heart and lung rehabilitation ward, an orthopedic rehabilitation ward, and a general elderly rehabilitation ward.

The researchers used the Revised Oral Assessment Guide (Renvert, 2003) stating that it had been reported to be a useful tool in detecting oral health problems among elderly ( $\geq 65$ years of age) rehabilitation patients. The investigators, however, did not provide additional information on the reliability or validity of the ROAG. There are eight categories evaluated in the ROAG. These categories are: (a) voice, (b) lips, (c) mucous membranes, (d) tongue, (e) gums, (f) teeth/dentures, (g) saliva, and (h) swallowing. Each category was described and rated on a 3-point ordinal scale from healthy (score 1) to severe oral health problem (score 3 ).

During the admission process, oral health problems were noted in $71 \%$ of patients. The most frequently reported problems were low salivary flow (56\%) and problems related to the lips $(50 \%)$. Significant associations were noted between low salivary flow and being a woman, living in special accommodation, and being undernourished. Additionally, the presence of respiratory diseases was associated significantly with findings in several of the oral assessment categories, with gum problems having the highest likelihood of having associated respiratory disease. 
The researchers found oral health problems in the majority of patients, regardless of the ward to which they were assigned. Associations between oral health problems and systemic diseases, especially respiratory disease, indicate the need for oral assessments to be a routine part of the hospital admission process. Results of this study suggest that implementation of a screening device such as the ROAG is beneficial in helping identify patients who will be at risk for serious systemic diseases. Since the oral cavity is typically not assessed during the standard hospital admission process, this study emphasized the need to make oral assessments a routine part of admitting elderly patients to a hospital. This finding underscores findings from the majority of similar studies regarding the importance of a pre-admission oral examination.

Stating that the dental care needs of those suffering from long-term illnesses have often been neglected, Sjögren and Nordström (2000) assessed the oral health status of this group. The researchers also addressed previous studies which indicated that hospitalized patients often have relatively poor dental health, and oral hygiene is adversely affected by living in an institution. The purposes of this study were (a) to describe the oral health status of patients in short-term and long-term care using an oral assessment guide and (b) to discover whether the use of such an assessment guide could distinguish any differences in oral health status between the two groups.

The investigators recruited the patients from four wards in the South-Eastern Stockholm Health District during the period of May to September 1994. Group A ( $n=$ 32) included patients from a general ward for acute and short-term care (i.e., less than 3 months). Group B ( $n=25)$ included patients from three wards for long-term care (i.e., more than 3 months). They, too, utilized the Oral Assessment Guide (OAG) used by 
Anderson, Hallberg, Lorefält, Unosson, and Renvert (2004), to collect information pertaining to each patient's oral health status.

The researchers found a statistically significant difference in the oral health status of Group A participants and Group B participants. Patients in long-term care (Group B) had significantly higher OAG scores, indicating poorer oral health, than patients in shortterm care (Group A). Additional significant differences between the 2 groups included the condition of the mucous membranes, gums, teeth, or dentures; the presence of calculus; and the appearance of the teeth.

Implications from this study underscore the importance of performing routine assessments of the oral health status of hospitalized patients, especially long-term patients. An assessment guide was shown to be a useful tool in estimating, planning for, implementing, and evaluating the oral care needs of patients.

Also interested in the oral health of people in long-term care facilities, Simons (2001) sought to determine the relationship between oral status and residents' requests for assistance with oral maintenance. According to Simons, in the past elderly people composed a relatively small portion of the British population, the majority of whom were edentulous and used dental care infrequently. Citing the National Diet and Nutrition Survey (National Diet and Nutrition Survey, 2008), Simons asserted that this demographic had changed, stating that half the British population age 65 and older are dentate. Simons also stated that there had not been any recent studies investigating the oral health practices of elderly people who live in residential homes. The purpose of this study, therefore, was to investigate the relationship between oral hygiene practices, 
requests for assistance with oral hygiene, and the oral health status of dentate elderly people living in residential homes.

The sample was obtained from a list of 110 residential homes in West Hertfordshire, England that provided long-term care for the elderly. A sample of 55 homes (50\%) was randomly chosen. A staff member from each home was contacted and was offered oral health examinations as well as access to dental services for all residents. From this large sample, 11 homes, with accommodations for 297 elderly people, were chosen. Only residents who were dentate, able to respond to a questionnaire, willing to provide a saliva sample, and give fully informed consent were subsequently invited to participate in the study. A total of 164 elderly people, $29(18 \%)$ males and $135(82 \%)$ females, participated.

Active root caries were found in $53 \%(n=87)$ of the subjects. Additionally, $32 \%$ of the residents $(n=52)$ had retained root tips. The male participants had significantly more retained roots than the females. The mean Plaque Index (PI) and Gingival Index (GI) were 2.3 and 1.6 , respectively, and were related significantly to the participant's age, coronal filled surfaces, coronal decayed surfaces, decayed root surfaces, and status of the denture. The mean PI and GI were also significantly related to low salivary flow rates, fewer teeth, and more filled root surfaces.

Regarding denture status, Simons (2001) found that the maxillary fitting surface had the poorest status and was significantly related to the levels of clinically diagnosed denture stomatitis, wearing dentures at night, number of retained roots, and salivary levels of yeasts and Streptococcus mutans. Questionnaire responses revealed that problems with eating, speaking, tasting, appearance, comfort, and looking after their own 
mouth increased with age in all residents. Nineteen percent $(n=31)$ of the residents expressed difficulty eating, which was significantly related to low salivary flow rates. Twenty-five percent $(n=41)$ of the residents had problems with taste which was significantly related to lower salivary flow rates, higher PI, and numbers of decayed coronal surfaces.

People who had teeth and dentures reported more problems speaking, tasting, and looking after their mouths than people who had teeth only. Subjects with decayed root surfaces had greater problems chewing and speaking, and requested more help with tooth cleaning, with significantly fewer people brushing their teeth twice daily. Only $20 \%$ ( $n=$ 15) of the residents without decayed root surfaces requested assistance compared to $44 \%$ $(n=38)$ of those with decayed root surfaces. The participants who were able to clean their mouths twice daily $(n=51)$ had significantly fewer yeasts, fewer filled root surfaces, lower PI and GI, fewer decayed root surfaces, and more sound root surfaces than those residents who brushed less often.

The results of Simons' study revealed significant problems of poor oral hygiene and lack of dental care for dentate elderly people. The positive relationships among oral hygiene, plaque, gingivitis, and untreated caries found in this study indicate that poor oral hygiene is a predisposing factor for the development of coronal and root caries in the elderly. This study indicated that a request for help in cleaning the mouth should be taken seriously by staff as perceived need for assistance was shown to be a good indicator of root caries and other dental diseases. Appropriate dental services to meet the needs of the elderly may involve trained oral care assistants rather than dentally qualified personnel. 
Also taking place in a long-term care facility, Montal, Tramini, Triay, and Valcarcel (2006) conducted a study with two distinct purposes. The first purpose was to determine the oral treatment needs of residents of a geriatric institution in France. Second, the researchers determined if self-reported oral health status was a reliable indicator of the actual oral health of the elderly residents of a long-term care facility, since self-reported oral health needs are typically lower than actual treatment needs. Because poor oral status has been associated with a decline in overall health, it is important to have an accurate method to determine an individual's actual oral health status. This determination, according to Montal et al., is further complicated by the fact that, when an elderly person enters a nursing home or hospital, an examination of the oral structures is seldom included in the patient's in-depth medical evaluation.

The investigators employed a descriptive design for their study. The sample for the cross-sectional study was patients $(n=321)$ over the age of 65 who had been institutionalized for a long period. Patients who either refused to have an oral health status evaluation or where it was not possible to evaluate a patient's oral health status were not included in the study. The investigators, however, did not include information regarding the numbers of people who did not participate.

Montal et al. used a survey questionnaire and an examination of each patient to collect their data. Each patient completed a questionnaire and had a clinical exam performed by the same dentist. The questionnaire consisted of various demographic factors as well as factors related to oral and systemic pathologies. Additional information regarding medications, dysphagia, and loss of appetite was also obtained. The clinical 
exam gathered information on decayed, missing, and filled teeth; status of removable or fixed prostheses; oral and prosthetic hygiene; and presence of halitosis.

Results indicated no significant differences in oral hygiene status with respect to gender. Oral hygiene, however, was significantly related to the level of hygiene of any prosthetic appliance. Another significant finding was the relationship between autonomy and gender with men needing more assistance with oral care than women. Last, the researchers found a significant negative correlation between the number of carious coronal surfaces and the number of teeth remaining.

Of major importance in this study was that the determination of treatment needs by the dentist was not consistent with patient self-evaluation of oral needs. The examining dentist found that $52.9 \%$ of the patients who had reported that there were no dental needs present actually required prosthetic treatment. Pertaining to the second purpose of this study, this finding suggests that an individual's self-evaluation of dental needs is not a reliable tool for planning treatment in an elderly population. These investigators as well recommended that, when an elderly person enters a nursing home or hospital, an examination of the oral structures should be included.

In 2002, Wyatt described the medical and dental status of elderly Canadians $(N=$ 369) residing in long-term care hospitals. Citing evidence that hospitalized elderly individuals were at considerable risk of dental diseases and were the group most likely to experience greater barriers to dental care, Wyatt (2002a) investigated the impact of oral health on three interacting themes: comfort, hygiene, and overall health.

Methods used in Wyatt's study included collection of baseline information for 369 elderly dentate subjects enrolled in a longitudinal clinical trial testing the 
effectiveness of antimicrobial mouth rinses in protecting teeth from caries. A total of 39 long-term care hospitals located in the Vancouver area participated in this study. Five of the hospitals were extended care hospitals accounting for 63 subjects and 34 of the hospitals were intermediate care facilities accounting for 306 subjects. Hospital administrators were asked to identify potential subjects; these were people who were dentate, could tolerate an oral examination, and could use a mouth rinse without difficulty.

The mean age of the subjects was 83.9 years with $72.4 \%(n=267)$ older than 80 years of age. The mean number of prescription and non-prescription medications per resident was 3.4. Medications known to produce xerostomic side effects were taken by $78.4 \%(n=289)$ of the subjects. Regarding dental status, it was found that the mean number of teeth per subject was 16.4 with a mean of 7.0 in the maxilla and 9.4 in the mandible. Assessment of plaque score data revealed that $7.9 \%(n=29)$ of the subjects had clean mouths, as indicated by a plaque score of zero. More than one-quarter of the subjects $(n=96)$ had a plaque index score of at least two on more than one-half of their teeth. Referrals for dental care were made for $253(68.6 \%)$ of the residents. The majority of referrals were made for the treatment of dental caries $(n=244)$.

Since the subjects in this study were relatively healthy, Wyatt concluded that their dental health was better than typically found in elderly residents of long-term care facilities. Additionally, it was noted that the majority of subjects in this study had poor oral health even though almost half had received dental care within the past 5 years. In order to improve the oral health of the elderly, Wyatt noted the need for early 
intervention in the institutionalized elderly population to prevent more serious oral disease sequelae from occurring.

A companion piece to the article on the medical and dental status of elderly Canadians residing in long-term care hospitals focused more specifically on the dental caries status of these same individuals. This companion piece was also published by Wyatt (2002b).

All exposed coronal and root surfaces were scored as absent, sound, restored, or decayed. Nine surfaces were scored for each intact tooth. Teeth missing all of the coronal surfaces were scored as fractured and the five root surfaces were scored. The calculation of decayed, missing, and filled teeth (DMF) was based on nine surfaces for intact teeth and five surfaces for fractured teeth. The examining dentist also obtained salivary samples to determine the presence and numbers of Streptococcus mutans and Lactobacillus, bacteria that have been associated with the increased presence of coronal and root caries in elderly populations.

Twenty-three percent of the residents' remaining teeth were scored as carious. No significant relation was noted between tooth types or between jaws. Over $78 \%$ of the residents $(n=290)$ had at least one carious lesion; $186(50.4 \%)$ had coronal caries and $254(68.8 \%)$ had root caries. Wyatt noted a significant positive correlation between the number of carious coronal surfaces and the number of carious lesions on root surfaces. Wyatt also noted a significant positive correlation between the number of carious coronal surfaces and the number of teeth remaining. This finding seems to contradict the results of the study by Montal, Tramini, Triay, and Valcarcel (2006), which indicated a 
significant negative correlation between the number of carious coronal surfaces and the number of teeth remaining.

While there were no differences noted in the number of remaining teeth between residents living in extended care hospitals and intermediate care facilities, the extended care residents had significantly more carious coronal surfaces. Lactobacillus scores were significantly correlated with the number of carious coronal lesions, the number of carious root lesions, and the Plaque Index.

In summary, Wyatt (2002b) found that residents of long-term care facilities have inadequate daily oral hygiene, high sugar intake, high incidences of caries, and a propensity for xerostomia; all of these results indicate extremely high caries susceptibility. Additionally, this demographic has the least access to oral care, primarily due to financial and transportation limitations. Wyatt found that prevention of caries in this population requires early intervention, education of health professionals in identifying at-risk individuals, and implementation of preventive programs. He also stated that prevention strategies may offer the most cost-effective means of controlling caries in the elderly population.

As noted in similar studies, (Andersson, Gustafsson, \& Buhlin, 2004; Nordström, 1990) the proportion of elderly people in the Swedish population is increasing. Because elderly people are often provided essential care at home, admission to a long-term care facility is often postponed. Consequently, patients admitted to a long-term care hospital are now older and more medically compromised than previously. Simultaneously, the improved oral health of the elderly population is reflected in the decreasing number of edentulous patients admitted to long-term care facilities (Andersson, Gustafsson, \& 
Buhlin, 2004). Citing studies noting that total edentulousness is significantly related to lower standards of dental care, Nordenram (1993) conducted a cross-sectional observational study. The purpose of the investigator's study was to determine whether changing concepts in community geriatric care and criteria for admission to long-term hospitals were reflected in the oral status of long-term hospital patients.

Nordenram stated that the dental staffs of the eight participating long-term hospitals were informed about the aim and design of the study, but she did not mention how the eight facilities were selected nor did she mention the total number of patients at the hospitals. All patients who were registered at the hospital dental clinics in February $1988(n=257)$ and April $1990(n=258)$ were included. An investigation sheet was completed from each patient's record including data regarding age, gender, case history, oral status, and current or planned oral treatment. The patients were classified in terms of their ability to undergo a dental examination. If a full examination including necessary radiographs and periodontal status was possible, the patient was described as "examinable." If full examination was precluded by physical or mental incapacity, the patient was classified as "partly examinable." If the patient was unable to participate and only a cursory examination to count the number of teeth was possible, the patient was described as "not examinable." The patients were also classified according to their treatment goals and their ability to tolerate treatment. The treatment goals were placed into one of four categories: (a) improve, (b) maintain, (c) postpone, or (d) relieve. The average age of the 1988 patients was 76.8 ; by 1990 the average age of the patients had increased to 79.1, although Nordenram does not mention if any of the individuals were the same in the two cohorts. The proportion of women had increased 
from $58 \%$ in 1988 to $69 \%$ in 1990 . The number of people in the age group 85 to 94 had increased by $8 \%$ while the group 74 years and below had decreased by $7 \%$. Again, by not mentioning if any of the individuals were the same in the two cohorts, it is difficult to determine if this demographic is a reflection of older people being admitted to long-term care or if it is merely normal aging of a cohort. In $1988,30 \%$ of the people were edentulous; by 1990 this percentage had decreased to $24 \%$ suggesting that, over time, older people are more likely to be dentate. Conversely, however, the mean number of remaining teeth was 15.2 in 1988; by 1990 this number bad fallen to 13.0. Although not stated in the article, this finding indicates that, while there were more dentate people in 1990 , the mean number of teeth was decreasing. Nordenram stated that gingival pockets deeper than $6 \mathrm{~mm}$ were recorded in 127 teeth (36\%) in 1998 and in 261 teeth (28\%) in 1990. Since the 1988 group and the 1990 group were very close in number, 257 people and 258 people respectively, it is unclear what the author means other than, while people were dentate, the corresponding periodontal health was worsening.

Regarding Nordenram's purpose, the oral status of long-term hospital patients had worsened from 1988 to 1990 . It would have been helpful if Nordenram had used a longitudinal model to more accurately classify the oral status of adults over time. Implications of this study suggest that it is important to have good oral health prior to entering a nursing home because of the rapid deterioration in status. Suggestions include pre-admission oral examinations and treatment so that care can be provided while the individual is in a healthier state. Once oral problems are noted in a long-term care facility, often the medical health is too compromised to permit any treatment other than palliative (Nordenram, 1993). 
Studies with similar purposes have taken place in Japan as well. Shimazaki, Soh, Koga, Miyazaki, and Takehara (2004) had two distinct purposes in conducting their prospective cohort study. The researchers were interested in determining the utilization of dental care by elders to establish an environment in which the elderly could receive dental treatment. Second, they studied the relationship between dental care and oral health to aid them in planning dental health projects for the aged.

Baseline examinations and interviews were performed on 1,929 (87\%) of the 2,220 residents of 29 institutions for the elderly in Kitakyushu, Japan between October 1988 and February 1999. A follow-up survey was carried out on the same subjects between October 1994 and March 1995. At follow-up, complete clinical and interview data were collected from 719 of the original 1,929 subjects; these are the data that were analyzed in this study. The oral health of each participant was assessed using mirrors, explorers, and periodontal probes. The examiners evaluated the status of caries, periodontal tissue, dentures, physical health, and mental bealth. Shimazaki et al. also asked the subjects about their desire for dental treatment at the baseline examination. Significant associations were revealed between dental care and physical and mental health status, presence of systemic diseases, number of teeth, and denture status. Dental care was significantly lower in the subjects who were age 80 or older, had fair or poor physical/mental status, had systemic diseases, were edentulous, had poor denture status, and had no desire for dental treatment at baseline. Interestingly, subjects who had no decayed teeth received dental treatment more often than the subjects who had one or more decayed teeth. Additionally, the fewer teeth the subjects had which needed to be extracted, the more dental treatment they received. Both at baseline and at follow-up, the 
treated subjects had more teeth and filled teeth and had fewer decayed teeth and teeth needing extraction than the untreated subjects (Shimazaki et al., 2004).

One of the purposes the investigators had was determining the utilization of dental care by elders to establish an environment in which the elderly can receive dental treatment. The examiners found that the more subjects needed treatment, the less they sought or received it. Second, regarding the relationship between dental care and oral health, the researchers found that dental treatment needs are reduced, even if only minimal care was provided. The authors concluded that dental treatment planning should take into account professional judgment as well as expressed desires of the elderly.

Stating that dental care of the institutionalized elderly was often limited to emergency care and not retaining teeth, Simunkovic, Boras, Panduric, and Zilic, (2005) sought to update information necessary in the planning of oral care for this population. The purpose of this study was to assess the incidence of dental caries and tooth loss in the elderly in Zagreb, Croatia.

The study was performed in a nursing home; a total of 139 subjects were selected independent of their dental status. Two examiners collected information on decayed, missing, and filled teeth (DMFT) as well as decayed root surfaces. The Community Periodontal Index of Treatment Needs (CPITN) was also determined for every participant. The examiners also recorded the presence of oral lesions, the status of any denture, how long it had been worn, as well as the need for other prosthetic treatment.

Of the 139 subjects, $108(77.7 \%)$ were female and $31(22.3 \%)$ were male. The average number of teeth with caries was 1.03 per person, the number of teeth that had been extracted was 6.9 per person and the number of teeth with fillings was 0.74 per 
person. The average number of teeth with root caries was 0.17 per person. Approximately nine teeth per person required some form of dental treatment. Significant DMFT differences were found between the age groups, with higher DMFT scores found in the older age groups, but they did not provide the relevant statistics.

Measurement of periodontal status revealed that only $18.7 \%(n=26)$ of subjects had healthy sextants, with calculus being present in $2.9 \%(n=4)$ of the subjects. Complete periodontal treatment was required in $5.8 \%(n=8)$ of the participants. Oral lesions were present in 73 of the participants with the most common site being the upper lip $(n=46)$ followed by the hard palate $(n=25)$, lower lip $(n=1)$, and the vestibule $(n=$ $1)$.

The examiners achieved their purpose of updating oral health information in order to more adequately plan dental care for this population. The results, however, are seemingly limited to this rather unique population. The incidence of past and present decay including root caries is quite a bit lower than has been reported in previous studies (Berkey \& Berg, 2001; Pla, 1994; Sjögren \& Nordström, 2000). This finding could be due to better oral care throughout the life span in Croatia, although the authors do not provide adequate epidemiological information to support that assumption.

Wardh, Hallberg, Berggren, Andersson, and Sōrenson (2000) investigated the attitudes of Swedish nursing staff to oral health care, in the hope that a deeper understanding would motivate further research in this area and possibly lead to better oral health care routines in the care of the elderly. The impetus for the study came from their assertion that, while the increasing number of natural teeth in older age groups is a sign 
of better general health, it is also a potential risk factor. Bacteria from teeth and periodontal tissues can constitute risk factors in frail individuals.

The participants in the study were 8 nursing assistants and 14 home-care aides from three centers for home care, two nursing homes, and two apartment homes for the demented elderly in central Sweden. Each interview focused on the subject's own description of assisting oral health care, on his/her thoughts, feelings, and actions in the situations described. The researchers stated that questions related to these areas were often raised spontaneously by the subject or were introduced by the interviewer in an informal and conversational way but did not provide additional information.

The investigators found that eight categories were grounded in the data. Four categories, (a) failing of knowledge, (b) failing of routines, (c) failing of support, and (d) increased workload, were related to a higher-order concept, labeled inner and outer circumstances for oral health care. The remaining four categories (a) violation of personal integrity, (b) abuse, (c) disgust, and (d) ethical dilemmas, were related to a second higher-order concept, labeled performance of oral health care.

Wardh et al. concluded that, based on 22 in-depth interviews with nursing staff working with elderly people, oral health care is given low priority. Further, the researchers found that, in theory, oral health care was considered important and most respondents thought that it ought to be done, but everyday problems and obligations interfered. Last, the investigators concluded that education in oral health care should include practical training in performing all sorts of tasks, however unpleasant.

In summary, international studies of oral health care of institutionalized adults revealed that oral health care is not a priority. This lack of an oral health priority was 
evidenced by findings revealing significant problems of poor oral hygiene and lack of dental care for dentate elderly people (Andersson et al. 2004; Nordenram, 1993;

Shimazaki et al., 2004; Simons, 2001; Simunkovic, Boras, Panduric, \& Zilic, 2005;

Wardh et al., 2000; Wyatt, 2002). To address this, however, Andersson et al. (2004)

found that an assessment guide may be a useful tool in estimating, planning for, implementing, and evaluating the oral care needs of patients. Additionally, Montal et al. (2006) found little difference in oral health status with respect to gender but did find that oral health status had a significant impact on the number of teeth remaining. In the following section, the investigators examine findings from studies performed in the United States.

\section{United States Findings}

It has been widely reported that an individual's oral health status deteriorates with age. This finding has been attributed to a number of factors including access to care (Adut, Mann, \& Sgan-Cohen, 2004; Schoenberg \& Gilbert 1998; Vargas, Dye, \& Hayes, 2002; Williams \& Butters, 1992; Wyatt, 2002), financial limitations (Williams \& Butters, 2002, Wyatt, 1992), and decreased ability to perform adequate oral hygiene procedures (Makhija et al. 2006). These findings are more pronounced among the population in a long-term care facility.

Citing numerous studies indicating that far greater numbers of people are now retaining their teeth to an advanced age, Sweeney, Shaw, Yip, and Bagg (1995) conducted a descriptive study to gather baseline information on the oral health of a large cohort of patients residing in a long-term geriatric hospital in southern Arizona. First, the investigators focused on increasing knowledge regarding the importance of disease of the 
dental hard tissues, especially root surface caries. Second, the authors discussed previous studies that demonstrated that, while elderly patients have significant oral health needs, there is little evidence of a coherent strategy for delivery of oral care to those in a longterm care facility. Third, the researchers also examined the delivery of oral care within the facility in addition to gathering baseline oral status information.

The study group comprised 77 female and 13 male hospitalized patients with a mean age of 83 years $(S D=8.0)$. All inpatients at the time of the study were included except for those who were too ill or confused to participate, although the investigators do not mention how many individuals this included. Forty-five (50\%) of the patients suffered from stroke and $22(24 \%)$ suffered from dementia. The remainder $(n=13)$ suffered from a wide range of disabling conditions.

Of the 90 patients, $46 \%(n=41)$ were entirely free of oral symptoms. The remaining $54 \%(n=49)$ all complained of at least one symptom. Regarding clinical appearance, the oral soft tissues of $40 \%(n=36)$ of the patients were clinically healthy. A total of 43 patients (48\%) exhibited oral mucosal pathology, while abnormalities of the dorsum of the tongue, particularly atrophic glossitis, were detected in $47 \%(n=42)$ of the patients. Overall, $60 \%(n=54)$ of the patients exhibited diseases of the soft oral tissues. Concerning status of the hard tissues, 11 individuals were dentate and caries was visible in $5(45 \%)$ of them. Out of the remaining individuals $(n=79), 73$ wore dentures. The examiners found that only three of the dentures fit well and were maintained adequately. Further, they found that only two individuals were free of denture stomatitis or cheilitis. At the time of the investigation, the hospital had no written policy on mouth care. The results of this study clearly demonstrated that improvement of oral health among the 
elderly institutionalized patients must become a priority in development of nursing standards and ward policies. The researchers suggest that, to address the significant oral health needs of elderly patients, a strategy for delivery of oral care to those in a long-term care facility must be developed.

Also interested in the oral health and hygiene status among residents of nursing homes, Murray, Ede-Nichols, and Garcia-Godoy (2006) measured the dental health status of elderly individuals residing in South Florida. Citing statistics that $20 \%$ of the United States population will be age 65 or older by the year 2030 , the researchers investigated the oral health status of a representative group of senior citizens to determine if an individual's level of oral health was related to age.

A non-invasive oral examination was performed on the residents $(N=265)$ of four nursing homes representative of socioeconomic diversity. Information gathered included (a) the prevalence of oral lesions, (b) levels of tooth loss, (c) oral hygiene scores, and (d) status of existing dentures. From these broader categories, one examiner further recorded (a) oral hygiene status, (b) numbers of remaining natural teeth, (c) prosthetics, (d) caries, (e) gingivitis, (f) tooth fracture, (g) xerostomia, (h) dysphasia or dsygeusia, and (i) the presence of soft tissue lesions.

The mean age of the residents was 77.3 years. Findings indicated that general oral hygiene scores and the level of calculus worsened as the mean age of the residents increased. Similarly, the mean number of teeth was also shown to be correlated with age with the mean age of edentulous patients being 81.4 years and the mean age of dentate patients being younger. The age of the patient was also shown to be correlated with the number of dentures worn, patients wearing one denture had a mean age of 77.4 and 
patients with two dentures had a mean age of 79.9 years. The investigators did not discover any findings related to the age of the residents and the presence of xerostomia and/or oral lesions.

As found in the previous study, results of this study indicated a need for a more comprehensive evaluation of nursing home residents to determine oral hygiene status and the incidence of untreated oral diseases. Additionally, the researchers recommended regular inspections of the oral status of nursing home residents so that necessary treatment can begin earlier and lessen the likelihood of the development of more serious sequelae. Of particular note is the finding that most residents aged 75 years and younger $(n=120)$ had 21-32 teeth while those aged 81 years and older $(n=54)$ were, on average, edentulous. This dramatic change in dentate status over 6 years highlights the need to ensure that a daily oral health care routine is provided by trained staff members in nursing homes.

Henry and Ceridan (1994) were interested in analyzing data from the National Nursing Home Survey (NNHS), to determine different characteristics of providing dental care to nursing home residents and community-dwelling older adults. Stating that the fastest-growing population is the age 85 years and older cohort, the authors estimated that by the year 2040, 4 million people age 65 and older will need nursing home care. The purpose of this study, therefore, was to determine differences in characteristics believed to be critical in the delivery of dental care to institutionalized and non-institutionalized elders.

Data for this study came from the 1985 NNHS, the 1984 National Health Interview Survey (NHIS) Supplement on Aging, and the 1987 United States Department 
of Health and Human Services National Institute of Dental Research (DHHS-NIDR) Survey. The sampling frame for the NNHS was based on approximately 14,000 nursing homes in the United States. The NHIS Supplement on Aging contained data from approximately 800,000 individuals, and the United States DHHS-NIDR Survey contained data from approximately 400,000 individuals. Henry and Ceridan (1994) analyzed these findings but did not provide additional details regarding collection of the original data.

Results regarding rate of edentulousness revealed that 50 to $77 \%$ of nursing home residents were edentulous, compared to $44 \%$ of homebound elderly, and $41.1 \%$ of community-dwelling elderly. Nursing home elderly had a mean of 18.6 decayed or filled (DFS) coronal surfaces and 6.5 DFS root surfaces. This was in sharp contrast to a mean of 5.1 DFS coronal surfaces and 1.5 DFS root surfaces of the homebound elderly and 5.2 DFS coronal surfaces and 3.2 DFS root surfaces of community-dwelling elderly individuals.

These findings are similar to those found by Sweeney, Shaw, Yip, and Bagg (1995) as well as Murray, Ede-Nichols, and Garcia-Godoy (2006) and call attention to the need for a comprehensive oral health examination prior to entering a long-term care facility. Additionally, this study characterized critical differences in oral health status of homebound elderly, individuals in nursing homes, and community dwelling elderly. Henry and Ceridan (1994) also underscored the number of obstacles and barriers that made providing dental care more difficult to this segment of the population. Finally, they stated that dental professionals must be committed to seeing that the oral health needs are met for all underserved populations in our society, especially the elderly. 
Also mentioning that the elderly population is quickly growing, Dye, Fisher, Yellowitz, Fryar, and Vargas (2007) analyzed the data from the 1997 NNHS to report oral health conditions and indicators of receipt of dental care for the institutionalized elderly population in the United States. The investigators stated that a goal of Healthy People 2010 (healthypeople.gov, 2008) was to include information on the receipt of dental care for nursing home residents aged 65 years and older and their analysis of the NNHS data would add knowledge to what was known about this population.

The 1997 NNHS was the fifth in a series of similar surveys conducted intermittently since 1973 . The sampling frame for the 1997 NNHS was based on approximately 17,900 nursing homes in the United States. The final analytical sample consisted of 1,488 facilities. Resident selection was performed with a random selection of up to six residents from each facility's roster yielding a final sample of 8,138 residents. Dye et al. (2007) limited their analyses to elderly residents, so their report contained information on approximately $91 \%(n=7,363)$ of all nursing home residents sampled.

The researchers found that $18.2 \%(n=1,325)$ of nursing home residents had received dental care within the past month. The percentage that received dental care was greater among those residents living in the Northeast (33.1\%) and the West (17.9\%) than in the South (12.9\%). This finding is similar to that found by Williams and Butters (2002). Among those aged 85 years or older, a greater percentage of those who were single or never married $(24.1 \%)$ at the time of admission had received dental care within the past month compared to those who were divorced, separated, widowed (18.1\%) or who were married $(16.2 \%)$. The percentage who received dental care in the past month was greater for dentate residents $(21.5 \%)$ than for edentulous residents $(15.9 \%)$. Last, 
concerning most recent dental visits, the investigators reported that a smaller percentage of residents received dental care in the past month if they were reported as having satisfactory condition of their teeth or gums $(17.4 \%)$ compared to those who reported having an unsatisfactory condition of their teeth or gums (23.9\%).

The researchers also looked at the size and nature of the facility in which the residents lived and found that a greater proportion of dentate persons who received dental care within the past month resided in facilities with 100 beds or more $(22.7 \%)$, had independent affiliation (24.4\%), or had nonprofit/government ownership (24.4\%). They also found that a greater proportion of individuals residing in facilities that employed a full-time dental professional received dental care in the past month (28.1\%) compared to those residing in facilities that did not employ a full-time dental professional (17.2\%). Last, regarding Activities of Daily Living (ADLs), the researchers found that more than $27 \%$ of residents with no ADL dependencies had received dental care within the past month as compared to those residents who had one or more ADL dependencies (16.9\%).

The investigators concluded that oral health issues in long-term care facilities would most likely remain a concern among geriatric dentists and dental public health specialists. A review of the data from the 1997 NNHS suggests that individuals residing in long-term care facilities are at an increased risk for decrements in oral health status potentially resulting in compromised systemic health.

Berkey and Berg (2001) also analyzed survey data to determine geriatric oral health issues in the United States. The Third National Health and Nutrition Survey (NHANES III) was part of a series of investigations conducted periodically to monitor 
health status in the United States. Conducted from 1988-1991, NHANES III documented, in part, dental health indicators for adults aged 18 to $75+$ years across the country.

While the researchers never mentioned the total number of subjects in NHANES III, they did say that among those surveyed, $71.5 \%$ of adults aged 65 to 74 years were considered dentate, with an average of 18.9 teeth. Of the adults older than $75,58.6 \%$ were considered dentate with a mean number of 16.1 teeth. The investigators also found that Hawaii had the lowest amount of edentulousness and southern states had the highest. This finding is similar to that found by Williams and Butters (1994). Not surprisingly, the number of decayed, missing, or filled surfaces (DMFS) increased with age. For adults in the 65 to 74 and $75+$ age groups, the researchers recorded mean DMFS values of 73.1 and 80.9 respectively. Approximately half the older adults had decayed, missing, or filled root surfaces (RDFS). Those in the 65 to 74 year age group had a mean number of 2.2 RDFS and individuals in the $75+$ age group had a mean of 3.1 RDFS.

Regarding periodontal status, Berkey and Berg (2001) found that $52 \%$ of those age 60 to 64 years presented with one or more markers of periodontal disease while only $40 \%$ of those in the 85 to 90 age cohort had evidence of periodontal disease. The authors did not state a reason for this finding but it may be plausible that individuals age 85 to 90 who remain dentate have probably exhibited good oral disease control throughout their lifetimes. The prevalence of attachment loss, however, increased with age. Attachment loss greater than $3 \mathrm{~mm}$ was detected in $35.7 \%$ of adults aged 30 to 39 years and involved $8.1 \%$ of teeth per person. By contrast, $89.2 \%$ of the adults in the 80 to 90 years cohort had attachment loss greater than $3 \mathrm{~mm}$ with $50 \%$ of the teeth being affected. The researchers also reported that furcation problems increased with age. In the youngest age 
cohort, furcation involvement was noted in $5.4 \%$ of individuals affecting only $2 \%$ of their molars. This prevalence jumped to $37.9 \%$ and $22.2 \%$ of teeth respectively among adults older than 80 years. Last, furcation problems most often involved the mandibular first molars in adults aged 56 to 90 years.

Findings from the review of NHANES III data included a clearer picture of the oral status of geriatric individuals in the United States. The researchers also recommended the encouragement of dental professionals to treat underserved populations through tuition incentive programs. Additionally, the investigators stated that schools that educate dental professionals should include an expanded geriatric dentistry curriculum to improve the likelihood of practicing professionals reaching out to elderly individuals.

Stating that older Americans have limited access to dental care due to economic barriers, Chiappelli et al. (2002) advocated the development of effective means that would identify and appropriate necessary treatment to these individuals. Further, the investigators stated that research evidence on the determinants of oral health status must be evaluated with respect to preventive and treatment services required to meet the oral health needs of older Americans.

Chiappelli et al. reported on data obtained from a nationwide survey of access to health care for adults $(N=7,265)$. Little information was provided on the collection of data, rather the focus of this article was analysis of existing data to identify various determinants of barriers to treatment. Additionally, the authors did not define "adults." This would have been helpful as many of the data referred to elderly individuals.

Regarding the importance of dental visits, the investigators reported that many elderly $(n=5,667)$ did not believe dental visits were necessary, particularly when they 
were edentulous. This finding was even more marked among those with lower income and less education. Further, Black and Hispanic elderly individuals were least likely to have had a dental visit within the past year. Chiappelli et al. found this particularly alarming because Hispanics in the United States comprise $11 \%$ of the population with Mexican-Americans in particular being the fastest growing ethnic group in the United States. Last, the researchers found that ethnic minorities generally had fewer preventive visits and more dental emergencies than white and middle-to-upper class respondents.

Findings from this study reiterate the importance of development of a policy ensuring that elderly individuals have access to dental care. Additionally, the investigators underscored the importance of continued studies of elderly ethnic individuals stating that they were most likely to have unmet dental needs.

In summary, literature reviews from studies performed in the United States revealed that elderly patients have a significantly increased likelihood of having poor oral health. This status was further compromised by various conditions including dementia, problems with activities of daily living (ADLs), and demographic factors including race and level of education.

In summary, regarding findings related to elderly people receiving institutional care in the United States, researchers (Dye et al., 2007) found that the type of long-term care facility had an effect on oral status of the elderly residents. Several investigators (Murray et al., 2006; Sweeney et al., 1995) reported that there was a close relation among denture status, oral hygiene status, and requests for assistance with oral care. Additionally, Henry and Ceridan (1994) found that institutionalized elders had a significantly higher rate of edentulousness than the homebound elders. While none of the 
authors reported a relationship between gender and oral status, all researchers found a significant relationship between the patients' ages and oral status. A recurring finding in all articles was the need to perform thorough oral examinations of patients prior to admission to a long-term care facility as an exacerbation of existing oral conditions was frequently noted following admission.

\section{Oral Health and Related Variables \\ Quality of Life}

Similar to demographic data concerning elders in most industrialized countries, (Steele, Pacza, \& Tennant, 2000; Sjögren \& Nordström, 2000; Isaksson \& Söderfeldt, 2007) the life span of Norwegians is increasing. As noted in other studies (Sjögren \& Nordström, 2000), the elderly in need of long-term care often have diseases that affect oral health. This diminished oral health, in turn, may affect quality of life. To address this, Henriksen, Ambjørnsen, Laske, and Axéll (2004) conducted a study with two purposes. First, the investigators were interested in oral hygiene, oral symptoms, and quality of life decrements among the elderly in Norway who were receiving long-term care. Second, they assessed the effect of physical and mental health on an elderly group's ability to receive comprehensive dental care.

Dental teams examined 1,910 adults aged 67 years or older who required longterm care. Seventy-one percent $(n=1,358)$ of the individuals lived in institutions and $29 \%(n=552)$ were homebound. Data were culled from the results of a standardized examination carried out between 1996 and 1997 by trained and calibrated dental teams. The examiners recorded the condition of the oral mucosa and oral hygiene using the Mucosal Score (MS) and Plaque Score (PS); the sum of MS and PS was then labeled 
MPS. Oral symptoms of the participants were ascertained from the answers to three questions concerning pain, chewing difficulties, and xerostomia. Each individual's perceived need for dental treatment was assessed by using a Treatment Ambition Inde: (TAI) although the investigators did not provide detail on the components of the TAl. The investigators found that the oral status (MPS) was significantly better in individuals who had dentures than in those who had only their own teeth. The MPS was also significantly higher in individuals who had their own teeth only and lived in institutions compared with individuals who lived in their own homes. Additionally, men had significantly higher MPS values than women. Oral symptoms were found in $13.4 \%$ of the participants $(n=182)$ and eating/chewing problems were reported by $31.4 \%(n=$ 600 ). Both parameters were most prevalent in individuals who wore dentures. Oral symptoms decreased with age as did eating problems. The researchers found the most prevalent TAI finding was "Limited Care" based on a "Fair" medical status; they found no significant associations between TAI and MPS.

In response to the first question posed by the investigators, this study indicated that many elderly adults requiring long-term care in Norway have unsatisfactory oral hygiene resulting in quality of life decrements. Regarding the second purpose of this study, the investigators found that the people most able to receive comprehensive dental care were those individuals with dentures. These findings indicated that there is a considerable surveillance and treatment challenge for this population (Henriksen et al.).

Penner and Timmons (2004) also sought to determine if there was any impact on the overall quality of life based upon the oral health status of senior citizens living in Prince Edward Island, Canada. They also explored the opinions and attitudes of seniors 
regarding their oral health status. Of special interest concerned findings regarding seniors who remain independent or were non-institutionalized. Citing growing evidence that, as the population ages, senior citizens are more likely to be healthier and remain in their own homes longer than previous generations, the researchers investigated the impact seniors' perceptions of oral health had on their overall quality of life.

The investigators used the Subjective Oral Health Indicators' Status (SOHIS) because they believed it was the most appropriate tool to measure the identified variables. The investigators also stated that test validity for the SOHIS had been previously established. Further, they claimed that the SOHIS was user-friendly in both readability and format, a matter of importance because most of the subjects had a low literacy level. The SOHIS scored subjects' responses on several topics (a) ability to chew, (b) ability to speak, (c) oral and facial pain symptoms, (d) other oral symptoms, (e) eating impact, (f) social relationships, (g) activities of daily living, and (h) worry/concern about oral health.

The research design incorporated a cluster sampling of public housing facilities for seniors; the clusters represented various geographic and cultural dispersions. Surveys were mailed to 547 of the 1000 seniors in residence (Penner \& Timmons).

More than half of the respondents (51\%) indicated that they had no teeth. The mean number of teeth was 6.44 and the mean age of the participants was 74.3 . The researchers did not, however, mention if the mean number of teeth was derived from only the dentate people or if edentulous people were also assessed. Regarding the question of quality of life and the ability to chew, the researchers found a significant association between difficulty in chewing and the number of teeth present. 
The second item measured by the SOHIS, ability to speak, indicated there was a relationship between this variable and age. Similarly, the concerns with oral/facial pain increased with age. The fourth item measured by the SOHIS, other oral symptoms, again correlated with age of the respondents. Dry mouth was the oral symptom most frequently noted, reported by $41 \%$ of the respondents.

There was also a significant association reported between the enjoyment of food/social concerns and age. Males scored higher in concerns related to the enjoyment of food while females expressed greater concern about the amount of time it took to finish a meal. The sixth item measured in the SOHIS concerned social relationships. The results indicated there was a statistically significant difference with regard to gender with males being more concerned about their oral health in social situations (Penner \& Timmons).

The impact of oral health on day-to-day living also was measured. Like many of the other dependent variables, this impact was correlated to age. Last, regarding worry/concern about oral health, this construct was correlated to the number of teeth present and age.

In regard to the first question posed by Penner and Timmons, it was determined that there was an impact on the overall quality of life based upon the oral health status of the senior citizens. They also explored the opinions and attitudes of seniors regarding their oral health status and found that the majority of respondents did not indicate a significant level of worry or concern. This study indicated the need for a more thorough assessment of the oral health of seniors. Since an association was shown between several 
factors related to age and/or gender, findings would be a useful tool in planning appropriate oral health care programs for an elderly population.

Also interested in assessing seniors' oral health-related quality of life (OHRQoL), Makhija et al. (2006) investigated a group of dentate and edentulous adults as part of a conceptual framework for the multidimensionality of oral health. The investigators addressed separate components of OHRQoL; these separate components were (a) oral disease and tissue damage, (b) pain and discomfort, (c) functional limitation, (d) oral disadvantage, and (e) self-rated oral health. Stating that numerous other studies had investigated the relationship between certain demographic factors and a decrement in oral health, Makhija et al. measured sociodemographic factors and OHRQoL stratified by dentate status in community-dwelling persons 65 years and older. The researchers, however, did not provide additional information regarding the reliability or validity of the instrument.

Subjects were recruited from participants in the University of Alabama Study of Aging, a longitudinal study of 1,000 community-dwelling older adults stratified by race, sex, and residence. Study of Aging participants came from a random sample of Medicare beneficiaries aged 65 and older living in one of five counties in central Alabama. Out of the 1,000 Study of Aging participants, 288 ultimately qualified to be in this study although the investigators did not provide additional detail on the potential participants who did not qualify. The stratification resulted in a dentate group $(n=203)$ and an edentulous group $(n=85)$. The researchers classified an individual as dentate if there were one or more teeth present; similar studies stratified groups more precisely according to dentate status (Makhija et al.). 
Of the OHRQoL decrements, there were three responses that were statistically significant between the dentate and edentulous groups. Thirty-three percent $(n=67)$ of dentate participants and $10 \%(n=8)$ of edentulous patients indicated their teeth looked bad. Five percent of subjects in the dentate group $(n=10)$ limited their contact with other people compared with none in the edentulous group. When asked about dental appearance, $34 \%$ of the dentate $(n=69)$ and $18 \%$ of the edentulous groups $(n=15)$ rated their dental appearance as fair or poor.

In the dentate group, the results also indicated that certain sociodemographic characteristics were strongly associated with OHRQoL. Participants with transportation difficulties, those with income less than $\$ 16,000 /$ year, African-Americans, and those with a 6th grade education or less were more likely to report OHRQoL decrements. There were fewer sociodemographic factors found to be significantly related to OHRQoL in the edentulous group. Although the investigators do not provide the data, they state that the only variables shown to be statistically significant to OHRQoL among edentulous people were race and an education level of grade 7 to 11.

The authors' recommendations from this study include the suggestion that geriatricians assess their patients' oral health status to increase the likelihood of a healthier aging. Further, although this study did not find significant dental relations between dental status and quality of life among edentulous people, the investigators suggested ascertaining the oral health status of all elderly individuals, not just the dentate segment of the population (Makhija et al.).

Reed, Broder, Jenkins, Spivack, and Janal (2006) also conducted a study that assessed oral health status and oral health-related quality of life (OHRQoL). The 
researchers stated that no studies had been identified that provided a simultaneous study of oral health quality of life, caregiver oral health education, and access to dental care. The investigators underscored the importance of this study by stating that the loss of functional independence may both impede a person's ability to access dental care outside of the institution as well as impair manual dexterity. There were two purposes of this study. First, the researchers examined oral health needs in a geriatric sample and related those needs to OHRQoL. Second, they wanted to assess and improve oral health knowledge among the caregivers at an extended care facility.

Reed et al. (2006) chose residents living in an urban extended care facility as the subjects for their study. In addition, employees at the facility participated in the oral health education seminars and oral health surveys. The researchers, however, did not provide information regarding how the particular facility was chosen nor the criteria for selecting the residents and employees for participation.

While the investigators did state that the study population comprised all consenting residents who were physically able to participate $(n=139)$, they did not provide any information regarding calibration attempts of the examiners. The authors conducted personal interviews with residents to collect information related to oral health behaviors and oral health quality of life assessments. Care providers were also given an oral health knowledge questionnaire pre-test specifically designed for the study project. Reed et al., however, did not provide information regarding the validity or reliability of the questionnaire.

The investigators determined that the mean length of time since the last dental visits of the residents was 1.3 years. Plaque was present in $61.9 \%(n=59)$ of the sample 
and calculus was present in $55.4 \%(n=53)$ of the sample. Each of these findings indicates poor hygiene among the majority of the sample. Other findings from the oral examinations include a mean of 2.4 diseased teeth, 20.1 missing teeth, and 1.2 filled teeth. Twenty-nine of the subjects were edentulous. The mean Decayed, Missing, Filled Teeth Index (DMFT) for this group was 23.8. The researchers stated that this was significantly greater than the mean DMFT reported for this age group (DMFT $=19.5)$ in the National Health and Nutrition Examination Survey III (NHANES III). The researchers, however, did not mention that NHANES III data represent the noninstitutionalized population.

Findings regarding the oral health knowledge questionnaires completed by the care providers $(n=18)$ indicated that oral health knowledge increased following educational in-service presentations. On average, care providers correctly answered $67.3 \%$ of the questions on the pre-test and $90 \%$ on the post-test indicating a significant improvement. The investigators stated that oral care providers attended additional workshops during which they observed and practiced oral hygiene techniques. Without conducting a follow-up examination of the residents, however, it is difficult to determine the effectiveness of the workshops.

Regarding the first purpose of this study, Reed et al. did examine oral health needs in a geriatric sample but they made no attempt to relate those needs to OHRQoL. Second, it appears that the researchers were successful in improving oral health knowledge among the caregivers at an extended care facility. It is difficult, however, to determine any long-term benefits of this improved oral health knowledge without reassessing individual residents. 
In summary, a review of literature concerning the association between oral health status and quality of life yielded several key findings. The investigators concluded that an individual's self-evaluation of quality of life was closely related with perceived and actual oral health status (Henriksen et al., 2004; Makhija et al., 2006; Penner \& Timmons, 2004). Additionally, several investigators noted that there was a strong correlation between quality of life and years of age; those who rated themselves as having a higher quality of life were the individuals who lived longest (Isaksson \& Söderfeldt, 2007; Reed et al., 2006). These studies again highlighted the need for a thorough oral evaluation to be performed prior to admission to an assisted living facility.

\section{Nutritional Status}

Lamy, Mojon, Kalykakis, Legrand, and Butz-Jorgensen (1999) explored the institutionalized elderly to determine the nature of the relation between poor oral health and nutritional status. Several factors have been shown to influence nutritional status, including general health, mental disorders, prescription drugs, socioeconomic factors, and oral health. Because the elderly are more likely to have poorer systemic health, use more prescription drugs, and have poorer oral health, the investigators believed that significant impact on nutritional status was likely.

The sample for their study was selected from a population of residents $(N=276)$ of eight nursing homes in Belgium. Subjects $(n=120)$ who met the selection criteria of age ( 65 or older), lack of cognitive impairment, and lack of acute illness were then selected. The examining dentist recorded numbers of remaining natural teeth, the type of prostheses, and the stability and retention of the prostheses (Lamy et al.) 
Subjects were classified into three groups based upon their oral status. The oral status groups were edentulous without dentures or with only one complete denture (Group 1), edentulous with two complete dentures (Group 2), and dentate with or without partial dentures (Group 3). The concentration of serum albumin, an indicator of undernourishment, was used to assess nutritional status. The subjects' nutritional levels were grouped into three categories: (a) patients with adequate nutrition, (b) patients at risk of undernourishment, and (c) patients who are undernourished.

The mean age of the subjects was 81 . Sixty-one percent of the subjects $(n=73)$ were edentulous and 15 of these subjects had no denture. Eleven of the edentulous subjects had one denture and 47 of them had two complete dentures. Dentate subjects had a mean number of 10.4 teeth. The mean nutritional score of Group 1 was significantly lower than in Group 2 and in Group 3. Subjects who could not perform the masticatory test $(n=63)$ had a significantly greater risk of undernourishment than subjects who were able to perform the test.

Results of this study indicated the need for comprehensive oral examinations of residents of long-term care facilities. Edentulous residents who wore at least one properly fitting denture had significantly higher nutritional levels than residents without prostheses. The investigators concluded that poor oral status places elderly institutionalized residents at a higher risk of undernourishment.

Soini et al. (2006) also explored the relationship between oral health and nutritional status in Helsinki, Finland. Frailty and malnutrition are common and interrelated items in the elderly and make elderly individuals more vulnerable for adverse health outcomes. The researchers' purpose was to determine the oral status of elderly 
residents in nursing homes and to describe the association between oral status and nutritional status.

The sample for this study consisted of residents of all 92 wards in the public and private nursing homes and all 53 long-term care wards in the Helsinki hospitals. Of the 2,424 public and private nursing home residents, $84 \%(n=2,036)$ participated in the study. Of the 1,444 patients in long-term care wards, $73 \%(n=1,052)$ participated in the study. Refusals were due to sickness, fatigue, or short length of stay.

Soini et al. classified oral status according to the presence and type of dentition and included the following categories: (a) natural teeth only, (b) mixed dentition (i.e., partial dentures with or without natural teeth), (c) complete dentures, and (d) edentulous with no prostheses. Oral health problems were categorized as follows: (a) chewing problem, (b) dysphagia, (c) oral pain, and (d) xerostomia. The ward nurses also collected data regarding nutrition related to the residents' eating habits and nutritional quality of the diet. The nutritional quality of the food was defined by three categories: (a) any food, (b) soft food, and (c) puréed or liquid food.

Results of the study (Soini et al.) revealed that the mean age of the nursing home residents was 83 and the mean age of the residents in long-term care wards was 81 . The investigators also discovered that most of the residents in nursing homes and long-term care wards were malnourished or at risk for malnutrition. Only $11 \%$ of the nursing home residents and $3 \%$ of long-term care ward residents were well nourished. Nutritional status was significantly associated with oral health problems with edentulous residents being malnourished or the group most at risk for malnutrition. 
Because the dentate residents were least likely to be malnourished or at risk for malnutrition, good dental care throughout the life span may reduce the likelihood of systemic sequelae as individuals age. Additionally, Soini et al. suggested that older adults are least likely to be consumers of dental services, underlying the importance of routine dental care as a portion of management of health care needs of seniors.

Citing studies suggesting that impaired chewing ability may adversely affect nutritional status and undermine general well-being (Henriksen, Ambjørnsen, Laske, \& Axél1, 2004; Penner \& Timmons, 2004), Takata et al. (2006) determined the impact chewing ability had on quality of life issues, including nutritional status. The authors' purpose was to determine if a correlation existed between ability to chew and a person's sense of well-being and satisfaction with daily living.

Participants for the study $(n=823)$ came from seven cities in the Fukuoka Prefecture of Japan. Takata, et al. (2006) used three questionnaires as their data-gathering instruments, but the third questionnaire scored nutrition status based upon ability to eat certain foods. To determine participants' scores regarding nutrition, each subject was asked about his/her ability to chew 15 foods. These 15 foods were selected to represent four food groups: three foods that are very hard to chew, six foods that are moderately hard to chew, three foods slightly hard to chew, and three foods easy to chew. The number of foods that each subject could chew was used as an index of chewing ability.

The mean number of teeth was 7.5 and the mean number of foods subjects could chew was 11.2. The authors noted a significant association between the number of foods individuals could chew and satisfaction with their physical condition. Similarly, there 
was a significant association between the number of foods individuals could chew and their satisfaction with social interactions.

An important finding from Takata et al. concerns the importance of tooth functionality as opposed to number of teeth. If one simply used the number of remaining teeth as an indicator of quality of life, the results could be misleading. The researchers suggested some measurement of mastication ability is an important component of an oral health assessment.

While several studies have examined the link between number of teeth and nutritional values, Nordström (1990) examined masticatory ability and the quality of dietary intake. The purpose of this study was to examine the relation between dietary intake and socio-medical background factors, dental status, and oral function.

Nordström's sample for this study came from a population of approximately 16,000 individuals in Umea, Sweden. Out of this population, 494 individuals were 70-79 years old. From this larger group, 183 people ( 94 men, 89 women) agreed to participate in the study. The investigation consisted of four main parts: (a) a socio-medical interview, (b) a medical study, (c) an oral study, and (d) a dietary study. The sociomedical interview included topics such as housing and living conditions, loneliness, and subjective assessment of health.

Nordström categorized dietary intake by developing four groups. Group A consisted of foods that provide energy, protein, fat, and thiamin. Group B consisted of Vitamin C-containing foods. Group C consisted of foods rich in calcium and iron, and Group D consisted of foods rich in Vitamin D. Results of this study revealed that women and individuals with subjective chewing problems had a significantly reduced intake of 
Dietary Group A, while people with less education had an increased intake. The author did not find a statistically significant predictor for intake of Dietary Group B. Females, reported mandibular dysfunction, and less education showed a negative influence on the consumption of foods from Dietary Group C, while stomach pain had a positive association with Dietary Group C. Last, Nordström reported greater age and mandibular dysfunction were significantly associated with a reduced intake of Dietary Group D.

Implications from this study include the need for regular assessment of the nutritional status of elderly populations. Additionally, the investigator suggested that further research is needed in this area to determine if specific nutrients could be supplemented to alleviate certain oral findings.

Also interested in the effects of oral health on eating and quality of life in an elderly population, Sheiham, et al. (2001) asserted that traditional oral epidemiological indicators are of limited use. While they are useful in assessing oral health status, traditional indicators do not provide information about the relationship between oral health and quality of life or the functioning of the oral cavity on the person as a whole. The purpose of this study, therefore, was to measure oral impacts that affect an elderly individual's daily life.

The sample for this study was a subset of participants in the British National Diet and Nutrition Survey of adults aged 65 and older. The survey used two separate population samples: one was a representative sample of the free-living population age 65 and older living in Great Britain; the other a representative sample of adults age 65 and older who were living in a long-term care facility. The oral health examination comprised a detailed dental examination including the numbet and condition of natural teeth and 
supporting tissues. Questionnaire content included the impact of oral status on daily living and choice of foods as affected by the teeth.

Sheiham, et al. examined a total of 753 non-institutionalized people and 202 residents of long-term care facilities. Of the free-living residents, 407 were dentate and 346 were edentate. The institutionalized sample was composed of 63 dentate and 139 edentate individuals. Overall, $13.7 \%$ (dentate) and $17.2 \%$ (edentate) of the free-living participants had one or more oral impacts on their daily life. For the institutionalized population, $31.8 \%$ of the dentate and $16.5 \%$ of the edentate population had one or more oral impacts on their daily life.

In the free-living group, fewer dentate individuals reported an oral impact; this trend, however, was reversed in the institutionalized group. In the free-living group, there was also variation in the prevalence of a dental impact according to the number of teeth; those with 1 to 10 natural teeth had the highest prevalence of impacts, whereas those with 21 or more teeth had the lowest prevalence of impact. The difference in oral impact between the dentate and edentate groups was not found to be significant in the institutionalized groups. The foods with which those with an eating impact most frequently could not eat or eat with some difficulty were apples, steak, raw carrots, nuts, toast, and lettuce.

Results of this study (Sheiham, et al.) indicate a need to assess oral impacts on nutrition for all elderly individuals. Since those individuals with more teeth had the fewest impacts on nutrition regardless of other variables, the emphasis of maintenance of a functional dentition is underscored. 
Stating that oral health status may be a particularly important factor for the nutrition of older people, Sheiham and Steele (2001) also conducted a study concerning the relation among dental status, the ability to eat certain foods, and nutritional status. The authors stated that both the volume and the quality of evidence directly linking oral health with nutrition have been limited. The purpose of this study, therefore, was to review the major findings from a large representative and comprehensive national survey in Britain, which assessed the degree to which the numbers of teeth and dentures affected older people's perceived ease of eating and their nutritional status.

The sample for the study consisted of a subset of individuals who had participated in the National Diet and Nutrition Survey of adults aged 65 and over, had completed an oral health interview, and undergone an oral examination. Sheiham and Steele divided the sample into two units; those individuals living on their own and those living in an institution on a full-time basis. In order to ascertain nutritional status, trained nurses collected blood and urine samples which were later analyzed.

The sample for the oral health survey was drawn from those individuals who had completed the 4-day dietary record. Sheiham and Steele reported that there were significant differences in the results for free-living people and those in institutions so the results were presented separately.

In the edentulous, free-living sample $(\mathrm{N}=346)$, difficulty eating apples, nuts, raw carrots, and tomatoes was reported. When compared with the dentate, free-living sample $(\mathrm{N}=407)$, the edentate group had significantly greater difficulty eating tomatoes, raw carrots, apples, nuts, lettuce, and well-done steaks. Among dentate participants, the number of natural teeth significantly affected the ability to eat certain foods. Concerning 
nutrient values, the edentate, free-living participants had significantly lower intakes of fiber, protein, calcium, riboflavin, niacin, Vitamin C, thiamin, and Vitamin E than the dentate population.

In the institutionalized sample, no significant differences were reported between the edentulous $(N=139)$ and the dentate $(\mathrm{N}=57)$ participants. The investigators reported, however, that there were significant differences between the free-living edentulous participants and the institutionalized edentulous.

Sheiham and Steele reported two findings of particular interest. The first was that people with 20 or more natural teeth consumed more of the majority of nutrients than people with fewer teeth. This provides further evidence that possession of more than 20 natural teeth is consistent with a good dietary capability and optimum nutritional intake. The second important finding was that fiber intake was much higher in people with more teeth and was significantly associated with the number of pairs of occluding posterior teeth. This study showed that the dental status of older people can have an impact on their ability to eat. This, in turn, was shown to affect food choice and preparation, and ultimately the intake and blood levels of some key nutrients. The investigators also provided rationale for increased education concerning the relationship between oral health status and nutrition.

Sheiham (2001) also conducted a study to determine the relationship among frequency and amount of sugar intake and caries. Stating that the relationship among mass, concentration, and frequency of sugar intake in free-living elderly individuals is strongly correlated to caries experience, Sheiham investigated the cariogenic capacity of 
specific foods to determine which were most likely to cause caries in an elderly population.

The sample for this study was a subset of participants in the British National Diet and Nutrition Survey (2000) of adults aged 65 and older. The survey used two separate population samples: one was a representative sample of the free-living population age 65 and older living in Great Britain; the other a representative sample of adults age 65 and older who were living in a long-term care facility. For this study, Sheiham (2001) focused on the free-living dentate elderly individuals because they were more likely than institutionalized individuals to be able to make decisions regarding diet. Out of 1,197 individuals who qualified for the study, 569 subjects kept a 30-day record of foods eaten and had undergone an oral examination. The diet record consisted of quantity of specific foods as well as the time they were consumed. During the oral examination, the researcher collected data regarding decayed, missing, and filled tooth (DMFT) and root surfaces (DMFR).

The mean age of the subjects was 81.3 , the mean DMFS was 16.3 , and the mean DMFR was 4.6. Sheiham categorized foods eaten into cariogenic and non-cariogenic. He further classified the consistencies of cariogenic foods into liquid, soft, sticky, and hard. The investigator found significant correlations between DMFS and the consumption of sticky and soft foods. The highest correlation was found between the consumption of sugared tea and DMFR. Last, concerning frequency of sugar exposure, Sheiham found that between-meal sugar consumption was significantly related to proximal caries.

Conclusions from this study include that the consumption of sugars, particularly sucrose, was the most important dietary cause of caries in an elderly population. 
Additionally, Sheiham concluded that the intake of extrinsic sugars beyond four times a day led to an increased risk of dental caries, especially root caries.

Schoenberg and Gilbert (1998) also addressed nutritional concerns of the over 65 age cohort but added that, within the older population, African-Americans represented the greatest number and proportion of minority elders, and their numbers were expected to rise dramatically over the next two decades. Further, the authors stated that the population of elderly African-Americans will have increased 121.2\% beyond their 1990 population, almost twice as much as the projected $64.7 \%$ increase among older whites. Additionally, compared to their white counterparts, African-Americans experience higher morbidity from heart disease, stroke, hypertension, diabetes mellitus, and arthritis. Oral health status may also influence food choices and may ultimately affect nutritional status. The researchers also stated that there was evidence that African-Americans have poorer oral health profiles than their white counterparts, including poorer chewing ability. Considering these various factors, the purpose of this study was to describe the prevalence of a broad range of oral health decrements that were believed to have dietary implications, and then to describe the association between these dietary decrements and selected characteristics, specifically race, poverty status, and educational level.

The goal of the sampling design (Schoenberg \& Gilbert) was to ensure that a large number of persons at an increased risk for caries and other oral health decrements would be included in the sample. High-risk groups of special interest were (a) lower income persons; (b) African-Americans; (c) residents of nural areas; and (d) persons who were 45 years old or older. Only persons with at least one remaining natural tooth were included 
in the sample. The investigators selected four counties in north Florida: three nonmetropolitan counties and one metropolitan county.

Telephone screening was used to identify a random sample of 5,254 subjects in households with telephones who (a) resided in one of the four counties of interest; (b) for the metropolitan county, resided in one of the urbanized zip codes; (c) were Englishspeaking; (d) were capable of engaging in a cogent telephone conversation; and (e) resided in a household, in contrast to a congregate facility. Of these subjects, 3,998 (76\%) had at least one remaining natural tooth. From the 3,998 subjects, a stratified random sample of 1,800 dentate subjects was selected for further study. Schoenberg and Gilbert (1998) then attempted to contact and recruit these 1,800 subjects by telephone. Of these, 873 subjects participated (48.5\%). The investigators conducted a baseline in-person interview of the 873 subjects, which was followed immediately by a clinical dental examination. Both the interview and clinical examination were used to gather information on oral health conditions that have dietary implications.

The in-person interview and clinical examination were used to obtain information about a broad range of oral health conditions. The clinical examination noted dental conditions that related to chewing ability, including (a) the presence and location of remaining teeth; (b) fractured dental restorations; (c) tooth fractures involving the cusp or incisal edge of the tooth; (d) tooth mobility; and (e) periodontal attachment loss. A separate assessment was also done for caries on the crown and root of each tooth. The researchers also ascertained self-reported measures of current oral disease and tissue damage. The authors assessed chewing ability by having subjects indicate whether or not they would be able to eat the following: (a) a whole fresh apple without cutting it; (b) 
steaks, chops, or firm meat; (c) fresh carrot or celery sticks; (d) fresh lettuce or spinach salad; and (e) boiled peas, carrots, green or yellow beans. Schoenberg and Gilbert measured oral health-related social and functional impact by asking subjects to report on whether dental, denture, or mouth problems caused them to avoid certain eating-related activities.

The mean age of subjects was 61.5 . The mean number of teeth present was 22.0 and was strongly associated with race, poverty status and education level. Forty-nine percent of subjects had 24 or more teeth, $30 \%$ had 17 to 23 teeth, $12 \%$ had 9 to 16 teeth, and $9 \%$ had 1 to 8 teeth. Although all subjects had at least one remaining natural tooth, $11 \%$ were edentulous in one arch.

The prevalence of root fragments, carious teeth, dental fractures, severely mobile teeth and severe periodontal disease was also substantial, and each of these conditions was significantly associated with race, poverty status, and education level. The prevalence of fractured fillings was $16 \%$ and was significantly associated with access to dental care, but was not significantly associated with race, poverty status, or education level. In the category of current oral pain, poor, less educated, and African-American respondents were significantly more likely to indicate the presence of current oral pain. However, self-rated current chewing ability was significantly associated with race, poverty status, and educational level. Only one of the measures, the percentage of subjects whose current oral health status prevented them from eating foods, was significantly associated with race and poverty status.

In addition to the presence of oral health decrements proposed to be related to dietary intake, Schoenberg and Gilbert provided evidence that African-American elders 
are at a heightened risk of poor oral health profiles, including such clinical measures as maintaining fewer teeth and being more likely to have a carious surface, fractured cusp or incisal edge, severely mobile teeth, and severe periodontal disease. African-American respondents more often indicated that they experienced tooth pain, at least one chewing difficulty, oral difficulties that prevented eating of certain foods, and dissatisfaction with chewing ability. These findings persisted regardless of poverty status or educational level, two factors commonly thought to confound racial differences in health outcomes.

To best remedy the greater risk to dietary intake faced by older AfricanAmericans requires understanding and addressing of all the many risk factors, including oral health. Despite the obviously greater need for prevention and treatment of oral disease, African-Americans reported seeking and receiving fewer dental services than whites.

Also interested in the oral health and nutritional status of elderly individuals, Pla (1994) determined which factors were significant in a decrement in either nutritional Status or oral health status. Stating that $40 \%$ of older Americans will require special oral health services based on complex health problems and functional status, Pla investigated the relation between nutrition and oral health in an institutionalized elderly population.

Subjects for the study came from a long-term care facility for the elderly in Washington, DC. Out of a total of 384 residents at the time of the study, 177 were dentate and agreed to participate in the study. Nutritional status was assessed by interviews with staff and individual residents. Subjects described how well they could hypothetically eat a variety of foods. Included in the list of foods were (a) raw carrots, (b) peanut butter, (c) steak, and (d) apples. Pla (1994) interviewed staff members to gather data concerning 
individual patient's diets as well as quality and quantity of food eaten. Nutritional status was then classified as "good," "fair," or "poor." The oral status of the subjects was ascertained by performing an intraoral examination. Each subject's oral cavity was assessed for coronal caries, root caries, gingivitis, and loss of periodontal attachment.

Pla found several significant relationships based on an individual's nutritional status. The subjects classified as having poor nutrition $(n=94)$ had significantly more coronal caries, root caries, and greater loss of periodontal attachment, than subjects classified as having fair $(n=62)$ or good $(n=21)$ nutritional status. No significant relations were found between nutritional status and gingivitis.

Pla concluded that primary care practitioners and/or screening health care professionals should always include an evaluation of oral status in assessment of an elderly person. Additionally, the authors suggested that an interdisciplinary team of physicians, nurses, nutrition professionals, dentists, dental hygienists, and social service professionals work together to ensure that good oral health status and adequate nutrition are maintained in older Americans. Finally, Pla concluded that public policy changes with regard to provision and funding of nutrition services would contribute to improving the health and quality of life for elders.

In summary, the preponderance of literature concerning an individual's oral health and nutritional status indicated that there was a strong and consistent relationship (Lamy, Mojon, Kalykakis, Legrand, \& Butz-Jorgensen, 1999; Nordström, 1990; Schoenberg \& Gilbert, 1998; Sheiham et al., 2001). Every study indicated that any one of various oral health decrements (particularly Nordström, 1990; Pla, 1994; Sheiham \& Steele, 2001; Soini et al, 2006, Takata et al., 2006) resulted in poorer nutritional status. 
Schoenberg and Gilbert (1998) further found that certain minorities were at greater risk for oral health decrements due to nutritional status. Because nutritional status is an essential part of overall health, the researchers concluded that oral examinations should be conducted on a regular basis for elderly individuals, especially those in a long-term care facility.

\section{Disabilities}

Saunders and Friedman (2007) investigated the oral health status of a group of elderly individuals who, while well enough to live at home, had disabilities which impacted their Activities of Daily Living (ADL). Stating that most studies of oral health and health care focused on either healthy, independent individuals, or individuals residing in nursing homes or other institutional settings, the researchers wanted to determine better the specific oral status of this population.

The investigators employed a convenience sample for this study. The subjects were obtained from a list of names given to the authors by one of 307 Medicareparticipating physicians in New York, Virginia, and Ohio. Ultimately, 641 subjects were chosen on the basis of age ( 65 or older), completion of an oral health questionnaire, difficulty in completing at least one ADL, and cognitive ability. A 42-item oral health questionnaire included questions on presence of teeth, xerostomia, burning mouth, jaw pain, perceived need for treatment, and utilization of dental services.

Approximately $55 \%$ of respondents $(n=353)$ reported fair or poor oral health status and $43 \%(n=276)$ reported they were edentulous. Sixty percent $(n=385)$ of the subjects reported xerostomia and 102 of these individuals reported a resulting dysphagia. Jaw pain was experienced by $5 \%(n=32)$ of the participants. Regarding burning tongue, 
$6 \%$ of the subjects $(n=38)$ reported they had experienced burning tongue occasionally with seven of the participants indicating they experienced burning tongue on a daily basis. Forty percent $(n=256)$ reported that they were currently in need of dental treatment while 269 individuals reported their last dental visit had been within the last 12 months.

Findings from this study suggest the importance of routine preventive care for all elderly members of a population. Stating that other segments of the elderly population are more clearly identified, Saunders and Friedman assert that there is a need for regular oral care for persons with disabilities.

Strayer (1993) was also interested in the oral health of the homebound elderly, especially those that considered themselves homebound due to limitations in ADL. He stated that, while the oral health of the institutionalized elderly has been reported to be poor, little information was available on the dental health of the homebound.

Additionally, he stated that the population growth of the elderly will present a unique challenge to dental professionals because the elderly are retaining natural teeth longer and experiencing increasing rates of dental disease, especially root surface caries.

Subjects for this study were clients of a social service agency that provided homebased services to functionally-dependent individuals. The subjects $(n=50)$, all volunteers, consented to a home-administered questionnaire and oral examination. Strayer stated that elderly individuals who considered themselves to be homebound ( $n=$ 30 ), would likely not have visited the dentist as recently as the non-homebound group ( $n$ $=20$ ) . Additionally, he stated that the homebound group would be more likely to report 
poorer dental health and perceive a greater need for dental care than the non-homebound group.

The homebound individuals had a mean of 15.0 teeth and the non-homebound individuals had a mean of 16.3 teeth. No significant differences were found between the two groups except for the number of home services received. The homebound group reported receiving a mean of 2.5 home services while the non-homebound group reported a mean of 1.6 home service visits. Dentate individuals comprised $56 \%$ of this population $(n=28)$ and had an average of 15.6 teeth per person. While not significant, the homebound group had a mean of 5.1 decayed or filled teeth and the non-homebound group had a mean of 7.3 decayed or filled teeth. This finding was different than what Strayer expected to find concerning the dental status of the homebound group. Perhaps, however, with a larger, more representative sample, findings would have supported his original hypothesis. Last, Strayer found a moderate correlation between general health and number of teeth with those individuals having more teeth reporting better perceived general health. Interestingly, individuals having more teeth also perceived a greater need for dental treatment.

Findings from this study highlight the need for health care providers and social service agencies to understand the changing scope of oral health needs and the limited access to dental care that the elderly cohort will experience. In light of the fact that numerous reports have documented the growing elderly population and the fact that they are retaining teeth much longer, tremendous demands will be placed on the current oral health care system. This information can provide guidance in the development of state and federal policies regarding delivery of dental care to the elderly population. 
In another study, hospital geriatric clinic patients $(n=230)$ were used to compare the oral health of individuals with dementia to that of persons with no dementing illness. Stating that there is little information on the oral health status of elderly individuals with dementia, Chiappelli, Edgerton, and Osterbrock (2005) gathered data on this population.

The researchers selected five long-term care facilities from northern California and patients whose families had consented to the study participated. Subjects and, when necessary, subjects' families completed a questionnaire regarding perceived oral health status. With the assistance of facility personnel, subjects were divided into those with dementia $(n=143)$ and those without dementing illness $(n=87)$.

The investigators found significant differences in the oral health status of the two groups. The group with dementia had significantly more gingivitis and root caries than the group without evidence of dementia. The group without dementia had significantly more restored teeth yet, surprisingly, significantly more teeth with furcation involvement. Such a finding may be in part due to a relatively small sample size although the researchers do not attempt to explain such a finding. Last, the authors did not find a significant difference in the relation between actual oral health status and perceived oral health status between the two groups.

Findings from this study underscore the importance of ensuring that all segments of the population have appropriate access to oral health care. Additionally, the investigators recommended that curricula of dental and dental hygiene schools be modified to include competencies regarding treatment of individuals with dementia, especially elderly individuals. 
In summary, elderly individuals with disabilities likely possess the greatest risk for decrements in oral status. The investigators (Chiappelli, Edgerton, \& Osterbrock, 2005; Saunders \& Friedman, 2007; Strayer, 1993) reported that disabled elderly people have significantly greater tooth decay and worsened periodontal status than individuals of similar demographic backgrounds without disabilities. Findings from these studies emphasize the need for individuals who provide health care services and implement public policy to understand the increased prevalence of serious oral conditions and the limited access to dental care experienced by elderly individuals with disabilities.

\section{Living in a Rural or Urban Area}

Williams and Butters (1992) analyzed the 1987 Kentucky Oral Health Survey to investigate sociodemographic factors of homebound people in Kentucky. There were two components to the study: a household interview and a clinical screening. For their study, an individual was defined as homebound if a physical or medical limitation made it unable to get out of the house more than 2 days per week.

Results from the survey indicated that $2.7 \%$ of households in Kentucky reported having a person who was homebound with the mean age of the homebound individual being 68.6 years. Additionally, households reporting a homebound individual had significantly lower household income than a household without a homebound person. There was, however, no significant difference found regarding urban or rural residence. While not statistically significant, analysis of the data revealed that $46 \%$ of households with a homebound individual reported spending no money on dental care during the previous year. This is in contrast to $22.8 \%$ of households without a homebound individual that reported spending no money on dental care during the previous year. 
Implications from this study include the importance of policy development regarding homebound individuals. Considering the fact that more than twice as many households with a homebound individual spent no money on dental services during the previous year, it is likely that serious oral health sequelae will be more pronounced in these individuals. Additionally, previous studies (Wyatt, 2002a) have noted the likelihood that the homebound population will increase in years to come, underscoring the need for appropriate public policy to be developed.

Investigating area of residence as a variable in oral health status, Adut, Mann, and Sgan-Cohen (2004) found a significant difference based upon geographic location. The investigators studied the oral status of the elderly population who attended adult day care centers in Israel. Participants in the adult day care programs were selected according to low-income status.

First, 11 centers were randomly selected from the list of 124 existing centers. The representative sample included centers in urban $(n=8)$ and rural $(n=3)$ areas. Second, a sample of subjects was randomly chosen within each center. The final sample $(n=338)$ included 110 males (32.5\%) and 228 females (67.5\%). Adut et al., however, did not provide information regarding how this sample was chosen.

Fifty-four percent $(n=182)$ of the sample was totally edentulous, among these $92 \%(n=168)$ wore full dentures, $5 \%(n=9)$ did not wear any dentures, and $2 \%(n=5)$ wore only one denture. The mean number of remaining teeth was 10.41 for the dentate population. The mean number of remaining teeth was significantly associated with education and was lower among people with no formal education than among those with 
high school education. No significant differences in the number of remaining teeth were found between people from urban populations and people from rural populations.

The authors also found no significant results in mean caries scores between people from urban populations and people from rural populations. Mean Decayed, Missing, Filled (DMF) scores were higher among those who did not seek dental care than among those who did. The investigators, however, did find that scores on the Root Caries Index (RCD) were positively associated with coronal caries. As for dental services utilization, the results indicate that almost $11 \%$ of older adults living in rural areas had never visited a dentist, compared to $1.4 \%$ of those living in urban areas.

The authors suggested that a community-based geriatric dentistry program should be implemented as part of all National Health Insurance Services. Needs among elderly who live in rural areas were found to be significantly greater and availability of dental services were lower than in urban areas. Recommendations include the fostering of a carefully implemented treatment plan of promotion, prevention, and early care to improve the oral health and quality of life of the elderly population.

Vargas, Dye, and Hayes (2002) examined data from several national surveys to describe differences among indicators of oral health status between rural and urban adults aged 18 to 64 years in the United States. The investigators stated that, although dated and sparse, existing information indicated that an oral health disparity existed between people living in rural and urban areas. Factors contributing to the disparity include greater distance to travel to be seen by a dentist and the lower dentist-to-person ratio existing in rural areas. Additionally, because rural adults have encountered greater barriers to 
accessing dental care, the assumptions of increased untreated dental diseases and poorer oral health status have been perpetuated.

Methodology for this study involved examination of data from several national studies: the National Health Interview Survey, or NHIS, from 1995, 1997, and 1998; and the Third National Health and Nutrition Examination Survey, or NHANES III, from 1988 to 1994. The overall sample size of the NHIS each year is approximately 110,000 participants representing more than 45,000 households. NHANES III collected sociodemographic and health data from personal interviews and examinations of the noninstitutionalized civilian population. The overall sample size of the NHANES III was approximately 38,000 participants. The investigators were most interested in the sociodemographic variable of rural/urban residence. For this study, Vargas, Dye, and Hayes used the U. S. Census Bureau's definition of nural as an area with fewer than 2,500 inhabitants.

Sociodemographic results indicated that rural residents were more likely to be poor than urban residents. The percentage of adults with private dental insurance was higher among those residing in urban areas. Regarding perceived oral health status, the researchers found that rural adults were more likely to report unmet dental care than were urban adults. The greatest percentages of adults who reported unmet dental needs were the rural poor. Rural adults were less likely to have had a dental visit in the past year than were urban adults. Additionally, adults residing in rural areas were more likely to have a greater caries experience as compared with those in urban areas. Last, adults aged 45 to 64 years of age residing in rural areas were almost twice as likely to be edentulous 
compared with adults in urban areas. The prevalence of edentulism was also significantly greater among rural adults than among urban adults stratified by poverty status.

Results from Vargas et al. indicated that the presence of edentulism among rural adults has important considerations for policy makers and practitioners. The investigators' conclusion that an important sociodemographic risk factor for tooth loss for adults aged 45 to 64 years of age was being poor and residing in a rural area indicated that greater emphasis needed to be placed on program planning for this group. Improving access to care and increasing dental care utilization in rural areas may require a renewed oral health care policy approach directed towards rural areas of the United States.

In 2003, Vargas, Yellowitz, and Hayes further analyzed data from NHIS and NHANES III to examine the oral health status and oral health care utilization of people 65 years of age or older who resided in rural areas. Citing evidence that the proportion of older people is increasing faster in rural areas than in urban areas, the researchers were interested in determining the magnitude of an oral health discrepancy to effectively plan intervention strategies.

As with the previous study (Vargas, Dye, \& Hayes, 2002), Vargas, Yellowitz, and Hayes (2003) analyzed the data from NHIS and NHANES III participants. Examining only those participants age 65 and older, the investigators analyzed the data of 24,457 NHIS participants and 6,002 NHANES $\amalg$ participants.

The researchers found that $67.6 \%(n=20,590)$ of participants had no dental insurance coverage, with those living in rural areas more likely to be uninsured than those in urban areas ( $72.1 \%$ and $66.1 \%$ respectively). Overall, $55.6 \%$ of elderly people reported having had a dental visit within the past year; those from rural areas were less likely to 
report having had a dental visit than those from urban areas ( $46.9 \%$ and $58.4 \%$, respectively). The researchers also analyzed indicators of perceived oral health status among people age 65 and older finding that $44.1 \%$ of dentate elders considered the status of their teeth to be poor, with rural residents being more likely to report poor oral status than urban residents (50.7\% and $42.2 \%$, respectively). Clinical oral health indicators were also examined. Regardless of place of residence, the investigators found that $18.2 \%$ of the participants had at least one untreated carious tooth. Additionally, there was no difference in the number of teeth with caries experience by place of residence. Last, the investigators found that the proportion of edentulous elders was higher among rural residents $(36.7 \%)$ than urban residents $(28.2 \%)$.

Findings from this study underscore the need for a comprehensive policy to address the disparity in health status based on place of residence. Factors important in addressing such a policy include the aging population and an increased tendency for elders to live in rural areas.

Stating that there are concerns about the capacity to provide adequate dental services to the aged, Adams, Slack-Smith, Larson, and O'Grady (2004) conducted a telephone survey to compare frequency of dental visits for individuals with urban, rural, or remote areas of residence. Specifically, they examined the proportion of older Western Australians who had visited a dentist within the past 12 months. Additionally, the authors were interested in providing a comprehensive description of dental visits by area of residence while documenting socio-economic status, oral health status, and the usual reason for a dental visit. Participants in the telephone survey were age 60 years or over, had a telephone listing, were on the electoral roll, and did not live in an institution. 
The investigators found that there was a significant association between the length of time since the last dental visit and place of residence (Adams et al., 2004). Urban residents $(n=766)$ were the most likely to have visited a dentist within the past year, followed by rural residents $(n=758)$, then remote residents $(n=494)$. Two-thirds of urban respondents $(n=500)$ reported a dental visit within the previous 12 months while over one-fifth $(n=262)$ of rural and remote residents had not been to a dentist in more than 5 years. Within each sex, age, income, occupation, and education group, the highest proportion of people having visited a dentist within the past year was in the urban areas and the lowest was in the remote areas. Self-reported prevalence of bleeding gums, loose teeth, or a recent toothache was not significantly different based on the area of residence. There were, however, significant differences in the reasons for a dental visit and in the difficulties associated with accessing services. Urban residents were significantly more likely to visit a dentist for a regular check-up than were rural and remote residents. The researchers also found that rural and remote residents reported significantly more difficulty in accessing dental care than urban residents.

After controlling for various factors, the investigators concluded that rural and remote participants were significantly less likely to utilize dental services regularly. Adams et al. (2004) underscored the importance of an increase in the numbers and accessibility of dental services to enhance utilization rates. Additionally, they suggested that the importance of regular oral health checks for the elderly needed to be widely promoted in the community and reinforced by other health professionals. As noted by others, however, the greater problem of maldistribution of health care personnel is also an 
issue that has likely resulted in the lower rates of utilization of health care services by individuals living in rural and remote areas.

Steele, Pacza, and Tennant (2000) also examined the effect of living in a rural community on availability of oral health providers. The purpose of their study was to examine population data and service provision data to analyze the difficulties faced by rural communities in seeking adequate dental care.

Population data were obtained from the 1996 census conducted by the Australian Bureau of Statistics. The authors collected data regarding the distribution of dentists from the Federal Branch of the Australian Dental Association using postal code information. To measure income status, the investigators used data sets from the Australian government describing the distribution of health care holders of all types.

Steele et al. found that, of the 264 postal code regions, $186(70 \%)$ had populations of fewer than 2,500 individuals. Additionally, most of these low-population regions were found in rural and remote areas. Of the total of 690 dentists who were analyzed in this study, the researchers found that the vast majority $(>85 \%)$ worked in postal code regions within major urban centers. Additionally, a total of 43 postal code regions (15\%) did not have a dental practice within their district. The investigators also found that, when the number of residents per dentist was distributed by postal code regions, an enormous disparity between rural and urban access to dental care became apparent. Last, the investigators found that the vast majority of individuals ( $81 \%$ ) with some form of health care coverage, resided in urban regions.

Although the researchers did not look at the oral health status of the residents living in rural areas, several other studies have identified rural place of residence as a 
factor in increased risk for poor oral health. The investigators did, however, underscore the importance of improving access for people living in rural areas.

In summary, the literature consistently revealed that rural individuals are more likely than urban individuals to have poorer oral status and a longer period of time between dental visits (Adams, Slack-Smith, Larson, \& O'Grady, 2004; Vargas, Dye, \& Hayes, 2002; Vargas, Yellowitz, \& Hayes, 2003). Additionally, tural individuals were significantly more likely to have an inaccurate perception of their oral status. Moreover, some authors suggested development of community-based programs or addressing public policy issues to improve access to care for the rural elderly (Adut, Mann, \& Sgan-Cohen, 2004; Steele, Pacza, \& Tennant, 2000; Williams \& Butters, 1992).

\section{Factors Related to Mortality}

Also interested in the oral status of elderly individuals in long-term care facilities, Ohrui et al. (2006) investigated the relation between dental status and mortality in Japan. As evident in the previously-mentioned studies, even though elderly populations are retaining their teeth due to heightened concerns about oral hygiene, Ohrui et al. stated that, while dentate, the elderly population has poor oral health. Moreover, in Japan, even though many institutionalized elderly people have lost many teeth, they do not use dentures to maintain their masticatory capacity. Such poor oral status of the institutionalized elderly may contribute to eating problems, poor nutrition, and an increase in intraoral bacteria. The purpose of this study was to analyze poor dental status as a risk factor for mortality.

Subjects were members of the Oral Care Study Cohort $(N=403)$, an ongoing longitudinal study in Japan. The participants were recruited from nine nursing homes and 
their mean age was 82.8 . The criteria for selection were that physical symptoms were stable for the preceding month and patients did not present with acute disorders such as pulmonary, cardiac, or neurological diseases. The investigators, however, did not provide information regarding the total number of potential subjects.

The patients were placed into three categories based on an evaluation of masticatory status. Group A consisted of patients $(n=99)$ whose dental status was functionally adequate for mastication with natural teeth only or natural teeth with partial dentures. Group B consisted of patients $(n=98)$ who were edentulous but kept their masticatory capacity by dentures in both arches. Group $C$ consisted of patients $(n=206)$ with a functionally inadequate dental status without dentures. There was no significant difference in the percentage of clinical conditions among the groups at baseline but the different levels of dental status were significantly associated with age (Ohrui et al.).

During the first 2-year follow-up, 112 patients died. There were 14 deaths $(12.5 \%)$ in Group A, $21(18.8 \%)$ in Group B, and $77(68.8 \%)$ in Group C. By the end of the 5-year follow-up, there were another 123 deaths. There were 45 deaths $(19.1 \%)$ in Group A, $54(23 \%)$ in Group B, and $136(57.9 \%)$ in Group C. The researchers also investigated the effect of dental status on mortality in an unadjusted analysis. As compared with Group A, Group C had a relative 2-year risk of mortality of 3.09 and a 5year risk of mortality of 1.93 . Group B did not have a significantly increased risk of mortality.

Regarding the investigators' purpose, an analysis of poor dental status as a risk factor for mortality, they determined that edentulousness was associated with approximately a twofold increase in the 2-year risk independent of age and gender. 
Findings from this study highlight a broader concern about inadequate dental status for mastication in institutionalized elderly people and its relation to poor outcomes. The researchers recommended systemic attention to dental status to minimize poor dental status-related deaths.

Citing reports that maintaining acceptable oral health status in subjects in need of long-term care is an urgent concern, Isaksson and Söderfeldt (2007) were interested in determining the current oral health status of a group of adults living in Sweden. The investigators were interested in having a clear concept of how to ascertain treatment needs to improve the efficiency in both screening and care of groups of elders residing in either permanent long-term care (LTC), nursing homes specifically for the elderly (NH), or individuals able to live on their own but with assistance for activities of daily living from ambulant nursing personnel or family members $(\mathrm{HC})$.

The study subjects were people within long-term care, in nursing homes, and in municipal housing who were examined by dental hygienists during their visiting oral health examinations during the year 2002. This group was observed for 2 years until the end of the study in 2004.

Dental hygienists responsible for the districts of the examined subjects performed the examinations in the subjects' homes. The hygienist assessed oral hygiene status using a Plaque Index (PI), oral mucosal inflammation using a Mucosal Index (MI), and oral mucosal friction using the Mucosal Friction Index (MFI). Based on these indexes, the treatment time needed for the dentist and/or hygienist was estimated for each subject (Isaksson \& Söderfeldt). 
There were 2,416 individuals examined at the initiation of the study in 2002; 1,170 of them had died by 2004 leaving 1,246 individuals. Only 914 of these individuals, however, were available for assessment; the results of this study are based on these 914 individuals. Regarding dentition, $36.6 \%$ of the individuals examined in 2002 had 10 or more teeth; in 2004 , however, $39.8 \%$ had 10 or more teeth. This finding is consistent with other studies (Ohrui et al., 2006; Holm-Pederson et al., 2005) and suggests that the number of natural teeth remaining is positively correlated with the life span. Two of the assessed variables revealed a significant impairment from 2002 to 2004 . These two variables were the Mucosal Index (MI) and the Mucosal Friction Index (MFI). The third assessed variable, the Plaque Index (PI), did not reveal any impairment over time. While the investigators did not state it, this could be explained by a temporary improvement in a subject's home care routine knowing the hygienist was coming to perform an assessment, whereas the results of the MI and MFI would not be affected by a recent improvement in home care. Results also indicated that $49.2 \%(n=450)$ of the population did not need to see a dentist in 2002 ; this number increased to $63.2 \%(n=578)$ in 2004 . This, too, suggests that subjects with the most need to see a dentist died before 2004 .

This longitudinal study supported much of what is known regarding the relation between oral health and life span. While this study did not specifically examine the impact of the number of teeth on life span, the results still are important in planning preventive measures for an elderly population. The researchers believe that their study should support the concept of "successful aging" including the retention of the natural dentition. 
Also interested in the connection between mortality and oral status, examiners in Stockholm, Sweden, sought to determine if there was a relation between systemic concerns including cardiac arrhythmias and dental concerns including caries and periodontal disease. Holm-Pederson et al. (2005) attempted to determine if a decline in the level of oral health was an accurate predictor of a general downturn in systemic health or if a decline in dental health was the result of worsening systemic health.

The population for the study $(n=129)$ was a group of community-dwelling people, age 80 and older in Stockholm, Sweden. The population was actually a subset of a much larger population $(N=1,810)$ involved in an ongoing, longitudinal, populationbased study that examined various characteristics of older adults including physical, psychological, medical, and social status. The 129 participants were chosen because of their dentate status and the fact that they were well enough to travel to and participate in a comprehensive oral health examination performed in a clinical setting.

Oral health data were collected in three parts: (a) completion of a structured questionnaire that a specially-trained dental assistant performed in a quiet environment, (b) completion of a self-reported oral health status survey, and (c) performance of a clinical oral examination. The questionnaire and self-reported oral health status survey measured various parameters of health including frequency of dental visits, selfperception of oral health, taste perception, and dietary habits. The clinical exam measured active coronal and root caries, periodontal pockets and clinical attachment loss, and exposed root surfaces (Holm-Pederson et al.).

While not statistically significant, the investigators did find that persons with active coronal caries were more likely to have cardiac arrhythmias than persons who did 
not. Results of this study, however, did reveal a statistically significant relationship between the presence of root caries and cardiac arrhythmias. Further, the magnitude of the root caries was shown to be a factor in the development of arrhythmias. Persons with three or more root caries had 2.5 times the likelihood of being diagnosed with an arrhythmia; the likelihood increased as the subjects got older (Holm-Pederson et al.). The presence or absence of periodontal disease was not shown to be a factor in the presence of cardiac arrhythmias.

Findings from this study suggest that there appears to be an association between cardiac arrhythmias and dental caries, especially root caries, in community-dwelling elderly. The hypothesis that root caries may be a predictor of a decline in health was also supported. Additionally, findings from this study support the need for oral assessments of elderly individuals in that discoveries related to an individual's systemic health may also be revealed. More research is indicated in that the subjects in this study were dentate and able to travel to the clinical site. It is likely that, because this study only measured dentate, relatively healthy individuals, findings from other individuals who were edentulous due to a history of periodontal disease were not included. The researchers acknowledge that a limitation of this study concerns the fact that there were no data regarding the cause of death from edentulous members of the population. Additionally, one could postulate that a decline in general health could be associated with a lessened ability to perform optimum oral hygiene procedures, thereby causing an increase in root caries. Further, the investigators did not address the role of the different bacteria associated with root caries and coronal caries. 
Hämäläinen, Meurman, Kauppinen, and Keskinen, (2005) were also interested in the relationship between dental status and mortality. Citing studies that demonstrate a statistically significant relationship between poor dental status and cardiovascular morbidity and mortality, the investigators designed a prospective cohort study of 85 -yearold Finnish individuals. The investigators had three purposes for conducting the study. First, they were interested in determining if acute oral infections would be associated with mortality. Second, they assessed whether the Community Periodontal Index of Treatment Needs (CPITN), number of remaining teeth, and urgent need of dental treatment were accurate predictors of mortality. Last, the researchers explored the correlations between blood test results and the parameters of general and oral health.

Subjects were participants in a population-based prospective study on the older residents of Jyväskylä, Finland. Baseline medical and dental examinations were conducted in 1995. Of the eligible population, $56.3 \%(n=94)$ took part in the medical examination and $55.7 \%(n=93)$ took part in the dental examination, although the investigators do not state whether the groups were mutually exclusive. The researchers calculated number of teeth and the CPITN as part of the baseline examination. As a portion of the CPITN, the investigators recorded data on gingival bleeding, calculus, and periodontal pockets. Edentulous subjects were regarded as free from periodontal infection and were given a score of 0 for the CPITN portion of the examination. A subject was regarded as being in urgent need of dental treatment if there was pain, infection, or the diagnosed condition was likely to have serious sequelae if left untreated.

The researchers stated that the medical examinations including blood tests were conducted by a physician familiar with geriatric problems. A disease was considered 
chronic if it had lasted longer than 3 months. The total number of chronic conditions diagnosed by the physician was used as a general indicator of morbidity. The number of years of full-time education was used as a marker of socio-economic status and self-rated health was determined by the participants' responses to a question asking them to describe their health during the last year.

In $1995,38.5 \%$ of the men $(n=11)$ and $59.7 \%$ of the women $(n=42)$ were edentulous. Of the dentate men and women $13.3 \%(n=2)$ and $24 \%(n=6)$ respectively had no need of periodontal treatment. Regardless of the number of teeth, $19.2 \%(n=5)$ and $13.4 \%(n=9)$ of the men and women were in urgent need of dental treatment. Acute myocardial infarction was the most common cause of death followed by septic infections and cancer. A significant correlation was found between the number of remaining teeth and the cause of death, with the majority of cardiac deaths recorded among the edentulous population. The researchers also found significant correlation between urgent need of dental treatment and cause of death although they do not provide further details. Similarly, the investigators stated that significant associations were noted between blood tests and parameters of oral health but no further details were provided.

For subjects in urgent need of dental treatment, the odds ratio for death was almost four times higher than those with no acute infection. Additionally, the results indicated that the lower the number of remaining teeth or the higher the number of chronic conditions, the higher the odds ratio was for mortality (Hämäläinen et al.).

Regarding the first purpose, the researchers did find an increased risk of mortality associated with oral infections. Likewise, they discovered that a poor score on the CPITN, a lower number of remaining teeth, and urgent need of dental treatment were also 
accurate predictors of mortality. Last, concerning the correlations between blood test results and the parameters of general and oral health, the investigators found significant associations.

The results of this study underscore the need for regular and frequent examinations of the oral structures of elderly individuals because oral infections may be fatal for frail elderly subjects. Further, it is important that such oral infections are diagnosed in a timely manner and eradicated as soon as possible in order to avoid severe health consequences.

A review of the articles regarding mortality revealed that various investigators (Hämäläinen et al., 2005; Isaksson \& Söderfeldt, 2007; Holm-Pederson et al., 2005; Ohrui et al., 2006) concluded that there was a significant association between oral status and mortality. This association was more pronounced when there was an urgent need of dental treatment. In summary, the investigators underscored the importance of regular oral examinations for elderly individuals because an increase in oral infections can be fatal for elderly individuals. Also, many factors contribute to the deterioration of an elderly individual's oral health status. Critical factors include access to care, financial limitations, and decreased ability to perform adequate oral hygiene procedures. The deterioration, in turn has been shown to affect nutritional status and quality of life attributes. It is critical that public policy be modified to address the oral status of elderly individuals, especially those in long-term care facilities and rural or remote areas.

Lastly, the relations of several variables to oral health status were explored. These variables were a) quality of life, b) nutritional status, c) presence of disabilities, d) living in a rural or urban area, and e) factors related to mortality. Common factors in these 
studies included the importance of optimum oral health throughout the lifespan and the critical need to establish policy to ensure that elderly individuals have access to appropriate oral care.

\section{Self-Perception of Oral Status}

Stating that elderly individuals are healthier today than in previous years, Andersson, Gustaffson, and Buhlin (2004) conducted a study to describe self-perceived oral health and function in a group of adults aged 75 to 84 years. Secondly, the authors were interested in determining the agreement between self-reported oral function and clinical findings.

The sample for this study comprised 150 randomly-selected individuals between 75 and 84 years of age. These individuals were selected from the total population ( $N=$ 2,910 ) of this age among residents of Huddinge, Sweden. The criterion for inclusion in the study was that the individual was able to live independently.

The investigators mailed a questionnaire and written information about the study to each participant. The participants for the clinical examination $(n=32)$ were randomly selected from among those who returned the questionnaire. The elements of the clinical examination were number of remaining teeth, presence of dentures, hygiene index, bleeding on probing, pocket depth, tooth mobility, presence of calculus, caries lesions, and xerostomia.

Results indicated that the mean age of the respondents was 78.7 years. Selfestimation of oral function revealed that most participants considered their chewing ability to be satisfactory. All participants indicated that they were able to chew cooked vegetables and $76 \%(n=98)$ claimed that they could bite into and chew raw carrots. 
Mouth dryness was perceived by $41.5 \%(n=58)$ of the participants and $11.8 \%(n=15)$ were aware of bleeding gums. The mean number of remaining teeth was 16 although 2 of the subjects were edentulous. Mouth dryness was noted by $47.8 \%$ of the respondents to the questionnaire, yet clinical examinations revealed $21.9 \%$ of the subsample had xerostomia. Bleeding gums were reported by $11.6 \%$ of the respondents to the questionnaire and by $12.5 \%$ of the subset that underwent clinical examinations. Statistical analyses disclosed no significant associations between chewing ability and xerostomia, bleeding gums, caries, calculus, or mobile teeth. There was good agreement among the total group of respondents to the questionnaire and those selected for clinical examination with respect to chewing ability and bleeding gums, but not for xerostomia.

As the number of dentate elderly in the community continues to increase, the importance of oral function to an individual's perception of general health and well-being is gradually emerging. The researchers demonstrated that perceived chewing ability and number of remaining teeth are related. One finding from this study, therefore, underscores the importance of access to appropriate oral care throughout an individual's life in order to maintain a functional dentition.

Also interested in the relationship between perceived oral health status and actual oral health status, Craig, Chu, and Bassett (2001) conducted a study with 29 elderly Canadians as participants. Citing statistics that little information is available on the dental health of the frail elderly living at home, the investigators' purpose was to determine the oral health status of a little-studied population of functionally-dependent elderly individuals living in a rural community. Additionally, the researchers investigated the perceived oral health status and dental health needs of the participants. 
The study was divided into two parts; completion of a questionnaire and participation in an oral assessment. The questionnaire was designed to obtain information regarding the participants' perceptions of their oral health status, their perceived need to see a dentist, their homebound status, the participant's oral health practices, and the participant's method of payment for dental care. Information gathered during the oral examinations included soft tissue pathology, xerostomia, periodontal pathology, oral cleanliness, abscesses, caries, and assessment of prostheses.

The ages of the participants ranged from 63 to 94 years with a median age of 80 . The participants' perception and rating of their oral health was similar to the examiners' findings in just over one-third $(n=10)$ of the cases. Forty-eight percent of the sample overrated their oral health status and $17 \%$ underrated it. Similarly, just over half $(n=16)$ correctly identified whether or not they needed to see a dentist. Forty-five percent $(n=$ 13) indicated that they did not perceive a need to see a dentist when a clinical need did in fact exist.

The oral assessments revealed that, of the 29 participants, $45 \%(n=13)$ were edentulous while $55 \%(n=16)$ had some natural teeth. Nineteen of the participants wore complete or partial dentures; it was discovered that $63 \%(n=12)$ of these individuals had ill-fitting dentures. Four participants never wore their dentures. The investigators determined that the need for dental care was high, as only $55 \%$ of the participants $(n=$ 16) reported visiting the dental office within the past 2 years and $35 \%(n=10)$ of the participants had not seen a dentist in over 10 years. Despite the fact that $86 \%(n=25)$ of participants reported regular brushing practices, the researchers found oral cleanliness to be generally inadequate. This finding was more pronounced among the dentate 
participants. Craig, Chu, and Bassett emphasized that, with the trend toward increased retention of the natural dentition into old age, oral hygiene is likely to be a major challenge.

The high need for dental care, infrequent dental visits, and poor oral hygiene put the elderly at risk of poor oral health and impaired general health. Additionally, the investigators suggested that lower perceived-than-actual need may be due to lower expectations for oral health by an older cohort. The fact that the majority of participants had ill-fitting dentures further suggests that oral adaptations by the frail elderly individual were coupled with low oral health expectations. The investigators demonstrated that oral health professions have important roles to play in ensuring the overall health and wellbeing of the functionally-dependent, community-dwelling elderly population.

Also concerned with the relation between self-perception of oral status and actual oral status, Westover (1999) conducted a study with rural Canadians as participants. He stated that a public health mandate was necessary to meet the needs of the entire population with a particular focus on segments that have a greater need. Westover, therefore, chose to study the oral status of elderly individuals residing in Western Canada, stating that much was known about urban Canadian and United States residents, but little was known about rural Canadian elders.

The survey consisted of two components, a questionnaire and an oral screening. There were 335 participants, representing $8.8 \%$ of the total population over 64 years of age, who participated in the questionnaire portion of the survey. For the oral assessment, 134 individuals agreed to participate, representing $3.5 \%$ of the total senior population. 
The questionnaire included sociodemographic information, perceived oral health status, preventive health behavior, risk factors, attitudes towards health, the use of dental care, and source of dental care. The screening measured edentulousness, decay, restorations, periodontal treatment needs, and mucosal lesions.

Westover reported that, of those with teeth $(n=67), 41.8 \%(n=28)$ had coronal caries averaging 0.88 caries per dentate person. Root caries were present in $14.9 \%(n=$ 10) of subjects with teeth, averaging 0.19 root caries per dentate person. There was an average of 5.04 teeth with coronal restorations and 0.62 teeth with root restorations per dentate person. Additionally, Westover reported that all respondents required periodontal treatment with $34 \%(n=23)$ requiring complex periodontal therapy. Of those that were completely edentulous $(n=67), 97.8 \%(n=66)$ had complete dentures. Regarding selfperception of oral health status, $76.4 \%(n=102)$ of respondents rated their dental health as somewhere between good and excellent.

The researcher found a discrepancy between self-reported oral health status and actual oral health status in that all dentate respondents had clinically-evident dental need. The majority of edentulous and partially edentulous respondents had calculus present on dentures and/or associated mucosal lesions. This information underscores the need for education regarding optimal oral health and appropriate use of dental services. Last, Westover (1999) concluded that many currently untreated conditions including coronal and root caries appeared to be due to a lack of preventive behavior and knowledge concerning optimal oral conditions. Educating this segment of the population regarding desirable oral health status would greatly improve oral conditions and, in turn, overall health 
Regarding perception of oral health status, a review of various studies revealed that correlation between actual oral health status and perceived oral health status is quite low (Andersson, Gustaffson, \& Buhlin, 2004, Craig, Chu, \& Bassett, 2001; Westover, 1999). This finding underscores the need to develop public policy which addresses access to health care for all individuals, especially the elderly.

\section{Summary}

This literature review has looked at the status of institutionalized patients, both internationally and in the United States; oral health and related variables including quality of life issues, the impact of nutritional status, the effect of the presence of disabilities, the effect of living in a rural or urban area, and oral health impairments related to mortality. Last, the literature review examined the relation between an individual's self-perception of oral status and actual oral status, a particular focus of the current study.

An overarching theme that emerged from this review of the literature was the need for development of public policy regarding oral health programs for the elderly. Several authors (Adams, Slack-Smith, Larson \& O'Grady, 2004; Vargas, Dye \& Hayes, 2002; Westover, 1999; Pla, 1994) stated that it was imperative for policy regarding oral inspections to be developed prior to nursing home admission. Others (Chiappelli, Edgerton, \& Osterbrock, 2005; Sweeney, Shaw, Yip \& Bagg, 1995; Vargas, Yellowitz, \& Hayes, 2003) supported the development of policy establishing regular oral inspections for institutionalized elderly.

The current study will incorporate what has been reported in the literature and further develop the relation between the oral health and various demographic factors associated with the elderly institutionalized residents of Kentucky. Specifically, the 
researcher will examine the accuracy of self-reported accounts of oral health status and the differences in the oral health of Kentuckians based upon area of residence prior to entering a long-term care facility, i.e., elders from areas defined as rural, urban, and small cities. 


\section{CHAPTER III}

\section{METHODOLOGY}

As noted in the previous chapter, there has been increased attention on the importance of oral health for all individuals. This emphasis on ideal oral health is even more important for elderly individuals because of findings linking poor oral health to heart disease and diabetes mellitus (Grossi \& Genco, 1998; Hujoel, Drangsholt, Spiekerman, \& DeRouen, 2000). Often, however, individuals are unaware of their actual oral health status (Andersson, Gustaffson, \& Buhlin, 2004) and delay seeking appropriate care. Additionally, numerous studies (e.g., Adams, Slack-Smith, Larson, \& O’Grady, 2004; Vargas, Dye, \& Hayes, 2002; Vargas, Yellowitz, \& Hayes, 2003) have found that individuals living in a rural community may be the group with the greatest decrements in oral health status.

\section{Oral Health of Kentuckians}

The most recent report by the Surgeon General (Oral Health in America, 2008) indicates that oral health in America is not adequate, especially among the aged. Additionally, Kentucky received a failing grade concerning adequacy of programs for elderly individuals (Oral Health in America, 2008). In order to begin to improve the oral health status of elderly Kentuckians, a state-wide oral health assessment was completed in 2005 to obtain a more accurate appraisal of actual oral health status. This assessment contained several critical elements: two of which will be the focus of this chapter; accuracy of self-reports and differences in oral health status based upon area of residence. 


\section{Kentucky Elder Oral Health Survey}

The Kentucky Elder Oral Health Survey (KEOHS) was a cross-sectional statewide oral health survey which assessed the oral health status and treatment needs of residents aged 65 and over. The KEOHS, conducted in 2005, included a questionnaire component (Appendix A) as well as a clinical component. The questionnaire portion of the KEOHS gathered information on demographics, general health questions, utilization of dental services, and self-reported oral health status.

The KEOHS gathered information on homebound residents, long-term care facility residents, and independently-living adults. To date, only data pertaining to the homebound residents have been analyzed. The focus of the current study, therefore, will be on data involving residents of long-term care facilities and assisted living facilities.

As part of the process for the 2005 assessment, a planning group consisting of representatives from the University of Kentucky's College of Dentistry, University of Louisville's School of Dentistry, the Oral Health Program for the Commonwealth of Kentucky, Area Agencies on Aging (AOA) for the Commonwealth of Kentucky, and senior centers and AOA regional offices across Kentucky were charged with developing a list of oral health markers that required assessments. Specifically, the survey was designed to compare results to Kentucky's previous oral health survey (Kentucky Cabinet for Human Resources, 1989) as well as to compare the oral health status of Kentuckians to people in other states and to national norms.

The questionnaire and clinical examination were pilot-tested at the LexingtonFayette County Senior Center and the Lexington Manor Nursing Home. A sample of 35 participants was included in the initial survey: 15 well elders at the senior center, 10 
residents of the nursing home, and 10 homebound elders registered through the Kentucky Area Agency on Aging. Each of the 35 subjects was interviewed using the planned survey instrument. Following the interview, subjects were questioned regarding the understandability of the interview questions. The survey was originally designed as a lengthy $70+$ item questionnaire that required more than an hour to complete. In order to make the survey more accurate and less difficult to complete, unnecessary questions, as well as those that were difficult to understand, were eliminated. Finally, the staff dentist conducted an oral examination on each of the 35 participants using the protocols that were planned to be used in the study.

The pilot test of the clinical examination resulted in the retention of the following markers of oral health: oral hygiene status, the presence of calculus, tooth mobility, gingival bleeding, recession, and caries. Approval for this study was granted by the Medical Institutional Review Board, Office of Research Integrity, University of Kentucky (IRB\#02-0182-F1V).

Subject Recruitment Methods

Reports from the 2005 KEOHS indicate that recruitment of elders was difficult. In the case of nursing home elders, the 54 certified assisted living facilities and 342 licensed nursing homes in Kentucky were contacted initially by phone and those agreeing to participate were sent a follow-up letter along with an explanation of the study and consent to participate. Next, a date and time were scheduled and the nursing home administrator, or his/her designee, was sent individual consent forms to be signed by the elders living in the nursing facility or the elder's designated care-giver so the elders could participate in the study. The benefit to the elder was that a free oral exam was provided 
along with the notion of contributing valuable information to the state. A total of 912 individuals from 27 nursing homes and assisted living facilities around the state agreed to participate in the study. Every elder who participated was given the questionnaire (or interviewed by the research team) and the clinical examination performed by a trained and calibrated dentist. The participant and nursing home administrator received a Dental Report Card with a summary of clinical findings of the participating resident along with the recommendation of when a dental visit was suggested. These recommendations ranged from a routine 6-month visit to as soon as possible.

Unfortunately, many of the nursing homes did not return calls, were concerned that the team was planning to report unfavorable information, or simply were not interested. Because of the difficulty with recruitment of nursing homes, instead of having a true, randomized sample of approximately $10 \%$ of the nursing homes in Kentucky, a convenience sample of nursing homes and assisted living facilities which agreed to participate comprised this aspect of the study's participants. Nursing homes and assisted living facilities that participated in the KEOHS are listed in Appendix B.

Information about each of the 912 participants included their area of residence and a zip code. Information from the U.S. Postal Service was used to determine if the individuals were from rural areas (towns with populations below 10,000 and other isolated rural areas), small cities (towns with populations between 10,000 and 49,999) and urban core areas (continuously built up areas of 50,000 persons or more; Zip Code Statistics, 2008). Analysis of zip code information revealed that 314 individuals came from areas defined as rural; 296 came from areas defined as small cities; and 302 came from areas defined as urban core areas (Table 1). Although there may have been 
significant differences in the 912 individuals in terms of income and education, the focus of the current study will concern place of residence prior to entering the long-term care facility as the independent variable affecting oral health status. Moreover, approximately $1 / 3$ of the participants came from areas defined as rural, $1 / 3$ came from areas defined as small cities, and $1 / 3$ came from areas defined as urban core areas.

Table 1

Place of Residence of Participants Prior To Entering Long-Term Care Facility

Number of Individuals

Rural

Small Cities

Urban Core Areas

314

296

302

Questionnaire

A questionnaire was created asking individuals to report items concerning overall oral health, missing teeth, gingival disease, periodontal disease, replacements for missing teeth, and adequacy of replacements. The questionnaire was developed in conjunction with the University of Kentucky's College of Dentistry; University of Louisville's School of Dentistry; Dr. James Cecil, Administrator of the Oral Health Program for the Commonwealth of Kentucky; Jerry Whitley, Director of the Area Agency on Aging (AAA) for the Commonwealth of Kentucky; and senior centers and AAA regional offices across Kentucky.

Self-Reported Oral Status

For the self-reported oral health status, each individual rated his/her oral health as excellent, good, fair, or poor. No criteria were provided, however, for the individuals to 
rate their health status. Additionally, participants reported frequency of tooth brushing and flossing, and the ability to chew a variety of foods. Following completion of the questionnaire, one dentist then performed a clinical examination on each of the 912 participants to rate each individual's oral health status. Data gathered by the examiner included oral hygiene status, and the presence of calculus; tooth mobility; gingival bleeding; recession; and caries.

\section{Clinical Determination of Oral Status}

The clinical oral health status rating was broken down into the same four categories as the self-report of oral status. The examiner, however, used the following criteria to determine the rating for actual oral status: a) excellent (excellent periodontal health, no caries); b) good (good periodontal health, four teeth or fewer with caries); c)

fair (fair periodontal health, five teeth or more with caries); and d) poor (poor periodontal health, and teeth with caries which are symptomatic or close to the pulp).

A person received a rating of excellent periodontal health if there was minimal recession and minimal gingival bleeding. A person received a rating of good periodontal health when there were one to two areas with recession and/or one to two areas of gingival bleeding. A person received a rating of fair periodontal health if there were three to four areas with recession and/or three to four areas of gingival bleeding. A person received a rating of poor periodontal health when there were five or more areas with recession and/or five or more areas of gingival bleeding.

\section{Statistical Analysis}

The methods of statistical analysis of the data detailed above included correlation statistics to determine the consistency of self-reported accounts of oral health status. In 
probability theory and statistics, a correlation (often measured as a correlation coefficient) indicates the strength and direction of a linear relationship between two random variables. It is very widely used in the sciences as a measure of the strength of linear dependence between two variables, giving a value somewhere between +1 and -1 inclusive. A value of 1 shows that a linear equation describes the relationship perfectly and positively, with all data points lying on the same line and with $Y$ increasing with $X$. A score of -1 shows that all data points lie on a single line but that $Y$ increases as $X$ decreases. A value of 0 shows that a linear model is not needed because there is no linear relationship between the variables (Gravetter \& Wallnau, 2007). Hypothesis 1, which stated that there will be a relationship between self-reported accounts and actual oral health status of elders, was tested by computation of the Pearson correlation coefficient. Each individual's self-reported status (excellent, good, fair, or poor) was correlated with the examiner's rating (excellent, good, fair, or poor) for agreement. It was expected that the correlation would be positive. That is, high self-report ratings of oral health should be associated with high ratings of actual oral health. Correlation coefficients would then be a useful data analysis tool in assessing the degree of consistency between self-report of oral health and actual oral health.

A one-way analysis of variance (ANOVA) was used to determine if there was a difference in an elderly individual's oral health status based upon place of residence prior to entering a long-term care facility. In statistics, ANOVA is a procedure in which the observed variance is partitioned into components due to different explanatory variables. ANOVA can be used to test the hypothesis that the means among two or more groups are equal, under the assumption that the sampled populations are normally distributed. 
Variation both within and between each of the groups is analyzed statistically, yielding what is known as an $F$ value. The $F$ value was then checked in a statistical table to determine if differences within and among groups are statistically different (Gravetter \& Wallnau, 2007). Hypothesis 2 , which stated that there will be a difference in oral health status based upon previous area of residence, was tested by analyzing examiner-rated oral health status (excellent, good, fair, or poor) by community type (rural area, small city, or urban core area) in which the individual resided prior to entering a long-term care facility. ANOVA will determine whether or not a statistically significant difference exists between the nursing home residents based upon this type of community in which they previously lived. The dependent variable will be the individual's oral health status and the independent variable will be the place of residence. It is expected that individuals from areas classified as rural or small city will have poorer actual oral health than individuals from areas classified as urban.

In summary, methodology for this study involved a more detailed analysis of archival data gathered during the 2005 KEOHS. In particular, data pertaining to the oral status of residents of long-term care facilities were analyzed. Specifically, the researcher examined whether a self-report is an accurate predictor of actual oral health status. Additionally, the researcher looked at whether the type of community in which an individual resided prior to entering a long-term care facility has any impact on oral status.

The following chapter details analysis of the relationship between actual oral health and self-reported oral health as well as the relationship between oral health status and the type of community. If it can be shown that a self-report is an accurate indicator of actual oral health, a community could more readily develop appropriate policy to ensure 
that those individuals in need of oral care could be identified and treated. Additionally, if the type of community in which an individual resided prior to entering a long-term care facility has an impact on oral status, policy could be developed to address the decrements in oral health status based on place of residence. 


\section{CHAPTER IV}

\section{RESULTS}

This chapter will examine findings from statistical analysis of the 2005 Kentucky Elder Oral Health Survey with respect to the two research questions. The first research question concerned the consistency between self-reported accounts of oral health status and examiner-reported accounts of oral health status. Hypothesis 1, which stated that there will be a relationship between self-reported accounts and actual oral health status of elders, was tested by computation of the Pearson correlation coefficient of these two variables. The second research question concerned differences in oral health status based upon the size of a community in which an individual lived prior to entering a long-term care facility. Hypothesis 2 , which stated that there will be a difference in oral health status based upon previous area of residence, was tested by analyzing examiner-rated oral health status (excellent, good, fair, or poor) by community type (rural area, small city, or urban core area) in which the individual resided prior to entering a long-term care facility.

As mentioned in the previous chapter, a total of 912 individuals from 27 nursing homes and assisted living facilities around the state agreed to participate in the study. Every elder who participated was given the questionnaire (or interviewed by the research team) and the clinical examination performed by a trained and calibrated dentist. Information about each of the 912 participants also included their date of birth, area of 
residence, a zip code, and various other demographic factors including income and level of education.

Closer inspection of the 2005 Kentucky Elder Oral Health Survey data revealed that there were multiple individuals with the same names. All but two individuals were able to be distinguished from one another due to other identifying factors. Every other participant was uniquely identified in some way. Subsequently, these two individuals were deleted from data analysis. Additionally, 4 individuals had scores on the examinerreported oral health status that were out of range. These four individuals were also deleted from data analysis because there was no way to determine their actual score. The final population, therefore, was a total of 906 individuals. Complete data were not available for all of the 906 individuals. The actual sample size varied by analysis and was dependent on which variables were analyzed.

\section{General Findings}

Participants in the 2005 Kentucky Elder Adult Oral Health Survey ranged in age from 65 to 103 years. There were 669 females and 237 males and the mean age of the 906 individuals was 81 years and 3 months. The mean clinician-reported oral health status was $2.27(1=$ excellent oral health, $2=$ good oral health, $3=$ fair oral health, $4=$ poor oral health) indicating an average oral health status between good and fair. Examining these findings by oral health status (Table 2), however, indicated that individuals with scores of 'fair' or 'poor' were older than individuals with scores of 'excellent' or 'good'. 
Table 2

Age by Oral Health Status

\begin{tabular}{lllll}
\hline & Excellent & Good & Fair & Poor \\
\hline $\begin{array}{l}\text { Mean Age of } \\
\text { Individuals }\end{array}$ & 80.11 & 79.71 & 82.09 & 83.06 \\
\hline
\end{tabular}

First Study Question

The first study question concerned the agreement between an individual's selfreported oral health status (excellent, good, fair, or poor) and the examiner evaluation of that same individual's oral health status (excellent, good, fair, or poor). Hypothesis 1 , which stated that there will be a relationship between self-reported accounts and actual oral health status of elders, was tested by computation of Pearson correlation coefficients. Out of the 906 participants, 832 individuals had completed a self-report and received an oral examination. Analyses revealed a significant correlation, $r=.23, p<0.01$, between an individual's self-reported oral status and the examiner-reported oral health status of the same individual. This correlation indicates that, although there was a positive correlation between a self-report and an individual's actual oral health status, the relationship was weak. Hypothesis 1, therefore, was accepted, indicating that there was a relationship between self-reported oral health status and clinician-reported oral health status.

Multiple Regression Analysis

The researcher was interested in a more in-depth analysis of this study question. Specifically, the researcher hypothesized that there may be differences in agreement 
between the self-reported oral health status and the examiner-reported oral health status based upon place of residence. In other words, did the accuracy of self-reports vary by region? This moderated multiple regression analysis revealed that there was a significant difference, $\Delta R^{2}=.008, F(2,826)=3.46, p<0.05$, in accuracy of self reports based on region (urban, small city, or rural). In order to determine which group or groups were most accurate, difference scores were computed between the clinician-rated oral health status and the self-rated oral health status by region. Analyses revealed that individuals from rural areas displayed the greatest difference in scores $(M=-0.36, S D=1.04)$ between the clinician-rated oral health status and the self-rated oral health status. Further, a mean score of -0.3636 indicates that rural individuals rated their oral health status higher than the clinicians. Individuals from small cities $(M=-0.06, S D=1.15)$ or urban areas $(M=0.04, S D=1.03)$ had difference scores of considerably less magnitude indicating that there was greater consistency between the clinician-rated oral health status and the self-rated oral health status.

\section{Second Study Question}

The second research question concerned the relation between the examinerreported oral health status and place of residence prior to entering a nursing home. Hypothesis 2, which stated that there will be a difference in oral health status based upon previous area of residence, was tested by analyzing examiner-rated oral health status (excellent, good, fair, or poor) by community type (rural area, small city, or urban core area) in which the individual resided prior to entering a long-term care facility. An independent samples $t$-test was executed to determine whether differences existed between the nursing home residents based upon the type of community in which they 
previously lived. The dependent variable was the individual's oral health status and the independent variable was the place of residence. The independent variable in the initial data set was recategorized from three variables (urban, rural, or small city) into two variables (urban v. non-urban) in order to answer the study question. The hypothesis for this study question was that individuals from areas classified as rural or small city will have poorer actual oral health than individuals from areas classified as urban. The reason for the recategorization was to be in agreement with the wording of the hypothesis, that individuals from urban areas would have better oral health than individuals from small cities or rural areas. Thus, the categories of urban and non-urban were developed.

Each individual's zip code was used to determine the type of community in which they resided prior to entering a long-term care facility. The mean oral health score for an individual who had previously resided in an urban area was $2.33(S D=0.97)$. The mean oral health score for an individual who had previously resided in a non-urban area (small city or rural area) was $2.22(S D=0.93)$. Analyses indicated that there was not a significant difference, $t(854)=1.58, p>0.05$, in oral health based upon place of residence prior to entering a nursing home. Hypothesis 2, therefore, was rejected, indicating that there was not a statistically significant difference in clinician-rated oral health status based upon living in an urban or non-urban area prior to entering a nursing home.

\section{Pearson Analysis}

The researcher was also interested in a more in-depth analysis of this study question. Specifically Pearson correlations were again performed to determine if there were differences in oral health status based upon an individual's income and level of 
education. Results of the first analysis revealed that income was weakly correlated, $r=$ $.139, p<0.01$, with oral health status. This finding is in agreement with similar studies that suggest there is a relation between income and oral health status (Vargas, Dye, \& Hayes, 2002). Results of the second analysis revealed that an individual's level of education was weakly correlated with oral health status, $r=-0.073, p<0.05$. Even though extremely weak, the negative correlation, however, indicates that as an individual's level of education increased, their clinician-rated oral health decreased. This finding, however, is not in agreement with similar studies that suggest there is a positive relation between an individual's level of education and oral health status (Vargas, Yellowitz, \& Hayes, 2003).

This chapter summarized the analysis of the data from individuals in long-term care facilities who had participated in the 2005 Kentucky Elder Adult Oral Health Survey. Results indicated that, in general, oral health status was worse for older individuals. Additionally, a weak correlation was found between self-reported oral health status and clinician-reported oral health status. Also, individuals from rural areas were found to display the greatest difference in scores between self-reported oral health status and clinician-reported oral health status. There were, however, no associations between clinician-reported oral health status and place of residence prior to entering a long-term care facility. Weak correlations were found between clinician-rated oral health status and the levels of income and education of the individuals.

The following section will examine how these results can be used to better plan programs for individuals in long-term care facilities. Specifically, if there are factors that 
can be shown to effect measurable change in the oral health status of older adults, appropriate programs should be developed to address the deficits. 


\section{CHAPTER V}

\section{DISCUSSION}

The focus of this chapter will be on how the analysis of the 2005 Kentucky Elder Oral Health Survey (KEOHS) can be used to make recommendations concerning appropriate oral health policy for elderly individuals. These findings are especially critical in light of literature which reports decrements in oral health status as we age (Andersson, Gustafsson, \& Buhlin, 2004; Simons, 2001). Additionally, these decrements are more pronounced among the individuals who reside in long-term care facilities (Andersson, Hallberg, Lorefailt, Unosson, \& Renvert, 2004; Montal, Tramini, Triay, \& Valcarcel, 2006). Last, individuals who resided in rural areas are especially at risk for poor oral health because of the limited availability of dental services (Adut, Mann, \& Sgan-Cohen, 2004).

Impetus for Changing Structure Paradigm

Since the first community began to artificially fluoridate its water supply in 1945 , many important milestones have been reached regarding the prevention of tooth decay. For many years, however, these oral health prevention programs have focused primarily on the needs of children. This group has benefitted from community water fluoridation, the application of pit and fissure sealants, and conservative tooth restoration to the extent that young adults today are much less likely to have had any teeth extracted due to rampant decay. This in turn has led to a remarkable drop in the rate of edentulousness in the United States (Table 3). In the 1950's the rate of edentulousness among people ages 
$55-64$ was $38 \%$, for people ages $65-74$ the rate was $56 \%$, and for people ages 75 and over the rate was $68 \%$. By the 1990 's, the rate of edentulousness among these same age groups was 20\%, 28\%, and 40\% respectively (Burt \& Eklund, 2005).

Table 3

Proportion of the U.S. Population Edentulous in 1957-58, 1971, 1985-86, and 1988-94 by Age

\begin{tabular}{lccc}
\hline & $55-64$ & $65-74$ & $75+$ \\
\hline $1957-58$ & $38 \%$ & $56 \%$ & $68 \%$ \\
1971 & $31 \%$ & $46 \%$ & $60 \%$ \\
$1985-86$ & $23 \%$ & $30 \%$ & $47 \%$ \\
$1988-94$ & $20 \%$ & $28 \%$ & $40 \%$ \\
\hline
\end{tabular}

Additionally, in 2008, legislation (KRS 156.160) was passed in the state of Kentucky which requires children to receive a dental screening before entering school. While these achievements are all certainly laudible, there has not been a corresponding emphasis placed on oral care for the elderly patient. A decrease in the rate of edentulousness, coupled with an increase in the average life span, has led to a population of elders that has remained dentate throughout their natural life. Unfortunately, however, many of these individuals lack the resources necessary to maintain optimum oral health. Even with adequate resources, most dental practices are not set up to care for the oral health needs of elderly patients. The primary focus in the majority of dental offices is on caries prevention and restorative procedures. The elderly patients often fall between the cracks because they typically do not require these services. Additionally, elderly people often have increased barriers including restricted transportation and limited income which 
make it more difficult to receive appropriate dental care services. These factors underscore the importance of restructuring the provision of dental care to the elderly in order to ensure healthier aging. Additionally, if an elderly individual is ultimately in need of residing in a long-term care facility, these factors may have contributed to less than ideal oral health.

First Research Question

Statistical analysis of the first study question revealed that a significant correlation existed between an individual's self-reported oral status and the examinerreported oral health status of the same individual. That correlation, however, was weak and indicates that self-reports may not be an appropriate measure of actual oral health status. Montal et al. (2006) found similar results when they reported that the determination of treatment needs by a dentist was not consistent with patient selfevaluation of oral needs. Likewise, results from the study conducted by Craig, Chu, and Bassett (2001) revealed that participants' perception and rating of their oral health was in agreement with the examiners' findings in only $34 \%$ of the cases. Westover (1999) conducted a comparable study and found a discrepancy between self-reported oral health status and actual oral health status in all dentate respondents. The only reported agreement between clinician-rated oral health status and self-rated oral health status was found by Andersson, Gustaffson, and Buhlin (2004). The researchers found that there was good agreement between the questionnaire and those selected for clinical examination with respect to chewing ability and bleeding gums. Unlike the Kentucky Elder Oral Health Survey however, the study by Andersson, Gustaffson, and Buhlin did 
not include having the participants rate their overall oral health status, rather just specific components were examined.

The weakness of the correlation found from analysis of the KEOHS data indicates that self-reports of oral health status should not be used as the sole oral health assessment in any pre-admission screenings. If a self-report of oral health status were a portion of the structure of pre-admission screening for a long-term care facility, however, an individual's self-report of oral status may either a) alert the facility's administrators that there is need for a dental follow-up or b) increase the awareness of the relation between oral health and systemic health.

Also critical is the finding that individuals from urban areas and small cities were significantly more likely to have an accurate appraisal of their oral health status than their counterparts from rural areas. Knowing the type of community in which an individual resided prior to entering a long-term care facility could help administrators plan more appropriate oral health care for residents of long-term care facilities if a self-report of oral health status was included in pre-admission health care appraisals. Since individuals from rural areas are less likely to have an accurate depiction of their actual oral health status, administrators should not rely on a self-report as a realistic depiction of oral health status. Until analysis of this aspect of the KEOHS data, no studies have been conducted regarding this research question. It had been reported that the agreement between self reports and clinician reports is weak. It has also been reported that individuals from nonurban areas have poorer oral health than individuals from urban areas. This study is the first to report on residence as a factor in accuracy of self-reported oral health status. 
Implications for practice from the first research question include ensuring that self-reported oral health status should not be the sole criterion for determining an elderly individual's oral health status. Additionally, even though individuals from urban areas were more likely to have a more accurate appraisal of their oral health status than nonurban individuals, an oral examination by a dental professional should be included as a routine portion of admittance to a long-term care facility.

Second Research Question

Results of the analysis regarding place of residence as a factor in oral health status revealed that there was not a significant difference in oral health based upon place of residence prior to entering a nursing home. This finding, however, is not supported by the literature. Vargas, Dye, and Hayes (2002) found that individuals from urban areas had better oral health than individuals from rural areas. The researchers suggested that this finding may be due to greater access to dental services. One possibility for the current study's finding indicating that there was not a significant difference could have to do with the fact that zip codes were used to determine the type of residence (rural, small city, or urban). An overarching assumption was that individuals from areas classified as rural would have greater limitations in access to adequate oral health care. While zip codes were an accurate means of determining area populations, they did not provide information regarding proximity to urban areas. As an example, there were 15 areas classified as rural based on population that were within 30 miles of Louisville. Similar rural populations existed close to Lexington, Elizabethtown, Owensboro, and Bowling Green. This could also explain why there was not a significant difference in the examiner-rated oral health status of individuals from various areas. 
A more in-depth look at this study question involved whether there were differences in oral health status based upon an individual's income and level of education. The preponderance of literature (Chiappelli, et al., 2002; Vargas, Dye, \& Hayes, 2002; Vargas, Yellowitz, \& Hayes, 2003) indicates that individuals with higher levels of education and greater income display better oral health than individuals with lower levels of education and income. Traditionally, income and education have been linked in the literature because of the close relation among these two variables and systemic and oral health. Findings from the current study indicated only a weak positive relation between clinician-rated oral health and income and a weak negative relation between clinician-rated oral health and education. These findings are so small, however, as to be almost meaningless. The finding regarding income and oral health status is widely supported in the literature (Makhija, et al., 2006; Schoenberg \& Gilbert, 1998). One explanation for the findings regarding the weak negative relation between education and oral health status could include the fact that many people with lower levels of education were likely edentulous and, therefore, not subject to a rating of 'poor periodontal health.'

Implications for practice from the second research question include determining not only an individual's place of residence prior to entering a long-term care facility, but determining proximity and availability of dental services as well. Even though an individual may have resided in an urban area, there may have been financial or other barriers to dental treatment. Similarly, an individual who had previously resided in a rural area may have had access to dental services in a nearby town. Place of residence alone 
should not be the sole criterion in assessing an individual's oral health status prior to admittance to a long-term care facility.

Implications for Practice

Implications from this study include the necessity to modify existing paradigms of dental practice, especially concerning the elderly. The current structure of dental practice in the United States is most appropriate for children, adults, and relatively healthy older individuals. The nature of a dental practice is such that patients must be ambulatory and in reasonably good health. Once an individual is no longer able to seek regular dental care due to physical limitations, financial limitations, or access to care issues, relatively few treatment options remain. Specifically, as outlined in Chapter 2, it is important to be aware of what resources are available in order to implement measurable change in the oral health status of older adults. In the situation involving elders living in long-term care facilities, appropriate modifications will need to come from outside the structure as internal resources are quite limited. Other countries have employed more appropriate models of oral health care delivery including visiting oral health specialists. Another example of such a modification in the current structure of oral health care delivery to elderly individuals could involve changes in policy regarding a minimal level of acceptable oral health prior to admission to a long-term care facility. Current admission standards for the majority of long-term care facilities do not mandate any oral assessment whatsoever. If a bill similar to KRS 156.160 was passed which required elderly individuals to achieve a minimum level of oral health prior to admittance to a long-term care facility, there would be increased awareness and impetus to treat the needs of this 
group. More importantly, however, decrements in oral health may be minimized if individuals were already at a specified level of oral health prior to admittance.

The impetus for KRS 156.160 resulted from the actions of individuals closely associated with elementary education. Educators were concerned over the number of missed school days due to oral health-related issues. The Kentucky Education Association contacted various legislators to encourage passage of a bill mandating oral health screenings for school children. A similar effort by the Kentucky Nursing Home Association would raise awareness of the plight of nursing home residents.

The preponderance of the literature reveals that older individuals have poor oral health. If a more appropriate structure for meeting treatment needs of the elderly can be developed, oral health outcomes can be vastly improved. This structure would include regular assessments of oral health status as well as a thorough oral examination prior to admittance to a long-term care facility. These structure improvements would naturally lead to improvements in outcomes: higher quality nutrition which, among other influences, affects quality of life and life span. This is especially important in light of several findings linking poor oral health and, subsequently, poor nutrition to earlier mortality (Holm-Pederson et al., 2005; Ohrui et al., 2006).

Implications for the Education of Practitioners

Additionally, dental hygiene schools must ensure that appropriate curriculum is in place to educate oral health practitioners on the unique needs of elderly patients. Currently, accreditation standards of the American Dental Association Commission on Dental Accreditation mandate that dental hygiene schools ensure that students are competent in treating geriatric patients. There is, however, no comparable standard 
regarding didactic content for the geriatric patient. Similarly, schools have a great deal of latitude in determining how many patients a student must treat to ensure that clinical competence has been attained. It is imperative that existing standards of dental hygiene education be modified to incorporate the changing nature of the elderly dental patient.

Additionally, accredited dental and dental hygiene programs in the United States have mandated competencies regarding community dental health classes. For several years, this researcher has guided dental hygiene students in planning preventive programs focused on improving the oral health of children. Given the focus of this project, however, future program planning activities will involve improving the oral health of the elderly.

Last, in 2001 the Kentucky Dental Practice Act was revised to include general supervision of dental hygienists. Patients eligible to be seen under the Dental Practice Act, however, must present with no serious systemic ajlments. Since it is unlikely that the majority of nursing home residents would meet these guidelines, it is imperative that the Kentucky Dental Association work with the Kentucky Nursing Home Association to develop policy that would allow these individuals to have regular access to oral health care.

\section{Significance}

The findings from this study underscore the need to ensure that appropriate policy be developed that includes oral health care for all segments of the population. Specifically, the finding regarding the deteriorating oral health status of individuals as they age underscores the importance of regular dental care for all aspects of the population. Significant improvements have been made in the oral health status of children 
in this country; largely due to increased attention and development of policy to ensure that access to oral care issues for children have been minimized or eliminated. Dramatic improvements in the rates of edentulism in the United States have led to an emerging new problem - oral care for the elderly. In previous years, most elderly individuals were edentulous and, therefore, not subject to many oral conditions faced by their dentate counterparts. It is important that appropriate policy be developed to ensure that healthy aging is possible for this group.

\section{Further Research}

There is a tremendous need to conduct a follow-up examination on the individuals in this study. Since each of the individuals had a thorough oral examination in 2005, conducting another examination in the near future would yield additional data concerning changes in oral health status. Especially important would be findings related to which individuals experienced greater decrements in oral health status as well as earlier mortality. If it were shown that individuals who had higher clinician-rated oral health status at admission maintained their oral health better than individuals with lower clinician-rated oral health status, there would be additional support for development of policy that mandated a minimal level of oral health at admission to a long-term care facility.

Additionally, it is important to compare results of the 2005 KEOHS to data collected in other states. Considering that the preponderance of literature suggests that oral health care for elders is a universal problem, merging of these data would provide a more accurate picture of the oral health status of individuals living in a long-term care 
facility. These results would be useful in providing even stronger impetus to modify the existing structure of dental care for elderly individuals.

One variable that would have been helpful in underscoring the need for radical change in the oral health care delivery system would concern nutritional status of the study population. Unfortunately, the 2005 KEOHS did not gather information on an individual's ability to eat certain foods or on an overall nutritional analysis. Since many studies have shown a positive correlation between oral health status and nutritional quality, this information would have been extremely enlightening. A modification in the questionnaire that was administered to the participants concerning the ability to eat certain foods would have been sufficient to provide additional valuable information.

The 2005 Kentucky Elder Oral Health Survey was a milestone in helping to raise awareness of the treatment needs of elderly individuals. Continued work in this field will result in improvements in the oral health status of elderly individuals. This, in turn, will have resulting effects on systemic health and, ultimately, quality of life. 


\section{REFERENCES}

Adams, C., Slack-Smith, L., Larson, A., \& O'Grady, M. (2004). Dental visits in older Western Australians: A comparison of urban, rural and remote residents. Australian Journal of Rural Health, 12, 143-149.

Adut, R., Mann, J., \& Sgan-Cohen, H. (2004). Past and present geographic location as oral health markers among older adults. Journal of Public Health Dentistry, $64,240-243$.

Andersson, K., Gustafsson, A., \& Buhlin, K. (2004). Self-perceived oral function in elderly residents in a suburban area of Stockholm, Sweden. Oral Health and Preventive Dentisiry, 2, 195-201.

Andersson, P., Hallberg, I., Lorefält, B., Unosson, M., \& Renvert, S. (2004). Oral health problems in elderly rehabilitation patients. International Journal of Dental Hygiene, $70-77$.

Berkey, D. \& Berg, R. (2001). Geriatric oral health issues in the United States. International Dental Journal, 5I, 254-264.

Behavioral Risk Factor Surveillance System. (2008, November 1). Retrieved November 3, 2008, from http://www.cdc.gov/brfss/

Burt, B. A. \& Eklund, S. A. (2005). Dentistry, dental practice, and the community. St. Louis: Elsevier Saunders. 
Chiappelli, F., Bauer, J., Spackman, S., Prolo, P., Edgerton, M., Armenian, C., et al. (2002). Dental needs of the elderly in the 21 st century. General Dentistry, 358363.

Chiappelli, F., Edgerton, M., \& Osterbrock, N. (2005). Dental needs of demented elderly. Gerontology, 24, 381-393.

Commission on Dental Accreditation. (2007, November). Accreditation standards for dental hygiene programs. Chicago: American Dental Association.

Craig, B. J., Chu, R. C., \& Bassett, S. (2001). Oral status of elderly receiving home support services. Probe, 35, 216-235.

Dye, B. A., Fisher, M. A., Yellowitz, J. A., Fryar, C. D., \& Vargas, C. M. (2007). Receipt of dental care, dental status and workforce in U.S. nursing homes: 1997 National Nursing Home Survey. Special Care Dentistry, 27, 177-186.

Gravetter, F. J. and Wallnau, L. B. (1999). Statistics for the behavioral sciences (5th ed.). New York: Brooks/Cole Publishing.

Grossi, S. G. \& Genco, R. J. (1998). Periodontal disease and diabetes mellitus: A twoway relationship. Annals of Periodontology, 3, 51-61.

Hämäläinen, P., Meurman, J., Kauppinen, M., \& Keskinen, M. (2005). Oral infections as predictors of mortality. Gerontology, 22, 151-157.

Healthy People 2010. Retrieved November 3, 2008 from http://www.healthypeople.gov/ Henriksen, B.M., Ambjørnsen, E., Laske, K., \& Axél, T.E. (2004). Oral hygiene and oral symptoms among the elderly in long-term care. Special Care Dentistry, 24, 254259. 
Henry, R. G. \& Ceridan, B. (1994). Delivering dental care to nursing home and homebound patients. Dental Clinics of North America, 38, 537-551.

Holm-Pederson, P., Avlund, K., Morse, D., Stoltze, K., Katz, R., Viitanen, M., et al. (2005). Dental caries, periodontal disease, and cardiac arrhythmias in communitydwelling older persons aged 80 and older: Is there a link? Journal of the American Geriatric Society, 430-437.

Hujoel, P. P., Drangsholt, M., Spiekerman, C., \& DeRouen, T. A. (2000). Periodontal disease and coronary heart disease risk. Journal of the American Medical Association, 284, 1406-1410.

Isaksson, R., \& Söderfeldt, B. (2007). Oral status and treatment needs among elderly within municipal long-term care 2002-2004. Swedish Dental Journal, 31, 45-52.

Kentucky Elder Oral Health Survey. (2005). Executive summary final report. Lexington: University of Kentucky Press.

Koop, C. E. (1993). Oral Health 2000. Second National Consortium Advance Program. Washington, D.C., February 12, 1993.

Lamy, M., Mojon, P., Kalykakis, G., Legrand, R., \& Butz-Jorgensen, E. (1999). Oral status and nutrition in the institutionalized elderly. Journal of Dentistry, 27, 443448.

Lopez, N. J., Smith, P. C., \& Gutierrez, J. (2002). Higher risk of preterm birth and low birth weight in women with periodontal disease. Journal of Dental Research, 81 , $58-63$. 
Makhija, S. K., Gilbert, G. H., Boykin, M. J., Litaker, M. S., Allman, R. M., Baker, P. S., et al. (2006). The relationship between sociodemographic factors and oral healthrelated quality of life in dentate and edentulous community-dwelling older adults. Journal of the American Geriatric Society, 54, 1701-1712.

Montal, S., Tramini, P., Triay, J., \& Valcarcel, J. (2006). Oral hygiene and the need for treatment of the dependent institutionalised elderly. Gerodontology, 23, 67-72.

Murray, P.E., Ede-Nichols, D., \& Garcia-Godoy, F. (2006). Oral health in Florida nursing homes. International Journal of Dental Hygiene, 4, 198-203.

National Diet and Nutrition Survey. Retrieved November 5, 2008 from www.food.gov.uk/science/dietarysurveys/ndnsdocuments

Nordenram, G. (1993). Changes in oral status and dental treatment in long-term hospital inmates in Stockholm from 1988 to 1990. Swedish Dental Journal, 17, 43-48.

Nordström, G. (1990). The impact of socio-medical factors and oral status on dietary intake in the eighth decade of life. Aging, 2, 371-385.

Ohrui, T., Matsui, T., Yoshida, M., Yoneyama, T., Adachi, M., Akagawa, Y., et al. (2006). Dental status and mortality in institutionalized elderly people. Geriatric Gerontology International, 6, 101-108.

Oral Health in America: A Report of the Surgeon General. Retrieved December 23, 2008 from http://silk.nih.gov/public/hck1ocv.@www.surgeon.fullrpt.pdf

Penner, A., \& Timmons, V. (2004). Seniors' attitudes: Oral health and the quality of life. International Journal of Dental Hygiene, 2, 2-7.

Pla, G. W. (1994). Oral health status and nutrition in old age. Primary Care, 21, 121133. 
Reed, R., Broder, H. L., Jenkins, G., Spivack, E., \& Janal, M. N. (2006). Oral health promotion among older persons and their care providers in a nursing home facility. Gerodontology, 23, 73-78.

Saunders, R., \& Friedman, B. (2007). Oral health conditions of community-dwelling cognitively intact elderly persons with disabilities. Gerodontology, 24, 67-76.

Schoenberg, N., \& Gilbert, G. (1998). Dietary implications of oral health decrements among African-American and white older adults. Ethnicity and Health, 3, 59-70.

Sheiham, A. (2001). Dietary effects on dental diseases. Public Health Nutrition, 4, 569591.

Sheiham, A. \& Steele, J., G. (2001). Does the condition of the mouth and teeth affect the ability to eat certain foods, nutrient and dietary intake and nutritional status amongst older people? Public Health Nutrition, 4, 797-803.

Sheiham, A., Steele, J. G., Marcenes, W., Tsakos, G., Finch, S., \& Walls, A.W.G. (2001). Prevalence of impacts of dental and oral disorders and their effects on eating among older people; a national survey in Great Britain. Community Dentistry and Oral Epidemiology, 29, 195-203.

Shimazaki, Y., Soh, I., Koga, T., Miyazaki, H., \& Takehara, T. (2004). Relationship between dental care and oral health in institutionalized elderly people in Japan. Journal of Oral Rehabilitation, 31, 837-842.

Simons, D., Brailsford, S., Kidd, E., \& Beighton, D. (2001). Relationship between oral hygiene practices and oral status in dentate elderly people living in residential homes. Community Dentistry and Oral Epidemiology, 29, 464-470 
Simunkovic, S. K., Boras, V. V., Panduric, J., Zilic, A. (2005). Oral health among institutionalized elderly in Zagreb, Croatia. Gerodontology, 22, 238-241.

Sjögren, R. \& Nordström, G. (2000). Oral health status of institutionalized patients. Journal of Clinical Nursing, 9, 632-638.

Soini, H., Muurinen, S., Routasalo, P., Sandelin, E., Savikko, N, Suominen, M., et al. (2006). Oral and nutritional status. The Journal of Nutrition, Health and Aging, 10, 495-501.

Steele, L., Pacza, T., \& Tennant, M. (2000). Rural and remote oral health, problems and models for improvement: A Western Australian perspective. Australian Journal of Rural Health, 8, 22-28.

Strayer, M. S. (1993). Dental health among homebound elderly. Journal of Public Health Dentistry, 53, 12-16.

Sweeney, M. P., Shaw, A., Yip, B., \& Bagg, J. (1995). Oral health in elderly patients. British Journal of Nursing, 20, 1204-1208.

Takata, Y., Ansai, T., Awano, S., Fukuhara, M., Sonoki, K., Wakisaka, M., et al. (2006). Chewing ability and quality of life in an 80-year old population. Journal of Oral Rehabilitation, 33, 330-334.

U. S. Census Bureau National Population Projections. Retrieved November 3, 2008 from http://www.census.gov/population/www/projections/summarytables.html

Vargas, C. M., Dye, B. A., \& Hayes, K.L. (2002). Oral health status of rural adults in the United States. Journal of the American Dental Association, 1672-1681. 
Vargas, C. M., Yellowitz, J. A., \& Hayes, K.L. (2003). Oral health status of older rural adults in the United States. Journal of the American Dental Association, 479486.

Wardh, I., Hallberg, L., Berggren, U., Andersson, L., \& Sörenson, S. (2000). Oral health care - a low priority in nursing: In-depth interviews with nursing staff. Scandinavian Journal of Caring Sciences, 14, 137-142.

Westover, W. (1999). Results of a seniors' oral health survey in rural Alberta. Probe, 33, $57-62$.

Williams, J. N., \& Butters, J.M. (1992). Sociodemographics of homebound people in Kentucky. Special Care in Dentistry, 12, 74-78.

Wyatt, C.C.L. (2002a). Elderly Canadians residing in long-term care hospitals: Part I. Medical and dental status. Journal of the Canadian Dental Association, 68, 353358.

Wyatt, C.C.L. (2002b). Elderly Canadians residing in long-term care hospitals: Part II. Dental caries status. Journal of the Canadian Dental Association, 68, 359-363. 
APPENDIX A

KENTUCKY ELDER ORAL HEALTH SURVEY

PARTICIPANT NAME INTERVIEWER

PARTICIPANT ID\#

INSTRUCTIONS: Please fill in or circle answers as appropriate. It is not necessary to answer any questions in this survey that you do not wish to answer.

\section{DEMOGRAPHIC INFORMATION}

1. What is your gender?

1 Male

2 Female

2. What is your date of birth?

$\frac{1}{\text { Month }} \frac{1}{\text { Date }} \frac{}{\text { Year }}$

3. In what town is your primary residence?

4. In what county is your home located?

5. What is your ZIP CODE?

6. What is your Area of Residence? Urban Rural 
7. Which of the following best describes your current living situation?

1 Home (house, apartment, mobile home, etc.)

2 Nursing home

3 Assisted living facility

4 Other

8 Don't know/Not sure

9 Refused

8. What is your marital status?

1 Never married

5 Married

2 Separated

8 Don't know/Not sure

3 Widowed

9 Refused

4 Divorced

9. What is your household composition?

1 Live alone

2 With spouse

3 With children

4 With relatives
5 With non-relatives

6 Live in Nursing Home/Assisted Living Facility

8 Don't know/Not sure

9 Refused

10. What is your race?

1 Asian/Pacific Island 5 White/Non-minority

2 American Indian/Alaskan Origin 8 Don't know/Not sure

3 Black/African American

9 Refused

4 Hispanic

11. What is the highest level of education (school) that you have completed?

Circle years of education completed, if you are currently in school, mark the highest level completed.

18 th grade or less

29 th - 12th grade

3 High school graduate or GED

4 Some college, no degree

5 Associate degree
6 Bachelor's degree

7 Graduate/Professional degree

8 Don't know/Not sure

9 Refused 


\section{Do you have any dental insurance that covers all or part of the cost of}

your dental treatment?

1 No dental insurance

2 Private dental insurance

3 Medicaid (any Medicaid recipient is eligible for some dental benefits)

4 Government pay program (such as Veteran's Administration)

8 Don't know/Not sure

9 Refused

Explanation of Dental Insurance: This is dental insurance only. This does not include general health insurance. This is insurance that covers you, not necessarily others in the family. "Private insurance" is traditional dental insurance such as Delta Dental or other private pay dental reimbursement plans. Medicaid is also known as KMAP, MA, MAP, The Medical Card, The Card, Medical Assistance, Kentucky Medical Assistance Plan.

Medicaid is not the same as Medicare.

Medicare is medical insurance for older (over 65) and disabled Americans. Medicare does not have any dental coverage. Medicaid does have some dental coverage.

13. Please give us an estimate of your family's income (for total annual household income).*

Circle only one response.

1 Less than $\$ 3,000$

$2 \$ 3,001-\$ 4,000$

$3 \$ 4,001-\$ 5,000$

$4 \$ 5,001-\$ 6,000$

$5 \$ 6,001-\$ 7,000$

$6 \$ 7,001-\$ 8,000$

$7 \$ 8,001-\$ 9,000$

$8 \$ 9,001-\$ 10,000$

$9 \$ 10,001-\$ 14,999$
$10 \$ 15,000-\$ 19,999$

$11 \$ 20,000-\$ 24,999$

$12 \$ 25,000-\$ 34,999$

$13 \$ 35,000-\$ 49,999$

$14 \$ 50,000-\$ 74,999$

$15 \$ 75,000$ and above

88 Don't know/Not sure

99 Refused

*Your best estimate of family income. From all sources including all family members living at home. 


\section{GENERAL HEALTH}

14. Would you rate your overall general health as being:
1 Excellent
3 Fair
2 Good
4 Poor

15. Please indicate health problems experienced during the past 12 months. Circle all that apply.

1 Anemia (low blood/iron)

2 Arthritis/Rheumatism

3 Back Problems

4 Cancer/Leukemia

5 Circulation Problems

6 Cirrhosis

7 Constipation

8 Diabetes (sugar)

9 Foot or nail problems

10 Gout

11 Heart Problems

12 High Blood Pressure

13 Injuries from fall/accident

14 Jaundice

15 Lung Problems
16 Osteoporosis (bone loss)

17 Paralysis

18 Parkinson's Disease (Palsy)

19 Prostate Enlargement

20 Recent Surgery

21 Shingles

22 Stroke

23 Urinary Tract Disorder

24 Vertigo (Dizziness)

$25 \mathrm{~TB}$

26 Hepatitis

27 Methicillin Resistant Staph Aureus

28 Other (specify)

29 None

88 Don't know/Not sure

99 Refused

16. Are you diabetic?

No Yes

Don't know/Not sure Refused

If Yes, how do you control your diabetes?

1 No control measures

2 Exercise and diet only (No medication)

3 Take pills

4 Use insulin (shots)
8 Don't know/Not sure

9 Refused

17. Are you taking any medication now?

No Yes Don't know/Not sure Refused

If Yes, how many medications are you taking?

Number of prescriptions Number of over-the-counter medications 
18. Do you regularly use mouthwash?

No Yes

Don't know/Not sure Refused

\section{If Yes:}

What brand do you use?

How many times a day?

19. Have any of the following conditions/diseases limited your mobility? Circle all that apply.

"Limited in mobility" means difficulty in going to church, doctor appointments, community centers, etc.

1 Stroke

2 Fall

3 Fracture (hip, knee, ankle)

4 Mental health (Explain:

5 Physical health (Explain:

6 Other

7 No limitations in mobility (SKIP TO QUESTION \#21)

8 Don't know/Not sure 9 Refused

20. If you have a limitation in mobility, how long has it lasted?

1 Less than 1 month 5 More than 1 year

$21-3$ months 6 Other

$34-6$ months 7 Not applicable

4 7-12 months 8 Don't know/Not sure

9 Refused

21. Are you able to feed, bathe, and toilet yourself?

Yes

No

If No, who is your primary caregiver?(the one who feeds, bathes and/or toilets you?).

1 Spouse 5 Caregiver in a nursing home or assisted living facility

2 Child

6 Other

3 Relative

8 Don't know/Not sure

4 Neighbor

9 Refused

These next 2 questions are asked to determine your risk for oral health problems and are not meant to be invasive in any way. Please answer as appropriate.

22. Regarding tobacco products:

22a. Do you smoke cigarettes every day, some days, or not at all?

1 Every day

8 Don't know/Not sure

2 Some days

9 Refused

3 Not at all (SKIP TO QUESTION \#22b) 
22a1. When you smoke, how many cigarettes a day do you smoke?

1 Less than 1 cigarette a day

2 1-3 cigarettes per day

3 4-19 cigarettes (less than 1 pack) per day

420 cigarettes ( 1 pack) per day

5 More than 20 cigarettes ( $>1$ pack) per day

8 Don't know/Not sure

9 Refused

22b. Do you now use spit tobacco (dip, snuff or chew) everyday, some days, or not at all?
1 Every day
8 Don't Know/Not Sure
2 Some days
9 Refused
3 Not at all (SKIP TO QUESTION \#23)

22b1. When you use spit tobacco, how many times a day do you use it?

1 Less than 1 time a day

8 Don't know/Not sure

2 1-3 times per day

9 Refused

3 4-19 times per day

4 More than 20 times per day

\section{Regarding alcohol:}

23a. Do you drink alcohol every day, some days, or not at all? $A$ drink of alcohol is I can or bottle of beer, 1 glass of wine, I can or bottle of a wine cooler, 1 cocktail, or 1 shot of liquor.

1 Every day

8 Don't know/Not sure

2 Some days

9 Refused

3 Not at all (SKIP TO QUESTION \#24)

23a1. When you drink alcohol, how much do you drink on the average per day?

1 Less than 1 drink per day 5 More than 6 drinks per day

21 drink per day

3 2-3 drinks per day

8 Don't know/Not sure

4 4-6 drinks per day

9 Refused

23a2. Considering all types of alcoholic beverages, how many times during the past 30 days did you have 5 or more drinks on one occasion? Number of times 8 Don't know/Not sure 9 Refused 


\section{ORAL FEALTH STATUS}

24. Would you rate your overall oral health (that is, the condition of your teeth and gums) as being:

If you have no teeth, then the rating is for your gums and other oral tissues
1 Excellent
3 Fair
2 Good
4 Poor

25. Do you regularly brush your teeth?

No Yes Don't know/Not sure Refused

If Yes, how many times per day? Circle only one answer.

1 1-2 times per day

2 3-4 times per day

35 or more times per day

If No, why not?

26. Do you regularly floss or clean between your teeth?

No Yes Don't know/Not sure Refused

If Yes, how many times per day? Circle only one answer.

1 1-2 times per day

2 3-4 times per day

35 or more times per day

If No, why not?

27. Overall, are you satisfied with: (whether you have natural teeth or have dentures, please answer).

a. your ability to chew any foods that you want?

Yes No Don't know/Not sure

b. your ability to speak clearly?

Yes No Don't know/Not sure

c. the appearance of your teeth?

Yes No

Don't know/Not sure

28. Do you have any current dental problems?

Yes No Don't know/Not sure

29. Do you have any pain in your teeth, gums or jaws?

Yes__ No____ (SKIP TO QUESTTON \#32 IF NO) Pain indicates pain in the teeth, gums, oral tissues, jaws, TMJ (jaw joint) or oral musculature. 
30. How long has your pain been present?

1 1 week or less $57-12$ months

2 Less than 1 month 6 More than 1 year

$31-3$ months 8 Refused

4 4-6 months 9 Don't know/Not sure

31. What type of dental pain do you have?

1 Pain in teeth 3 Pain in jaws (including jaw joint)

2 Pain in gums 4 Other (Explain)

32. Have you lost any natural teeth for any reason?

Yes No

If yes, how many teeth have you lost?

1 Minimal tooth loss ( 5 or less teeth)

2 Some or partial tooth loss (6 or more, but not all teeth)

3 No remaining teeth (SKIP TO QUESTION \#36)

33. How many of your teeth have been removed because of tooth decay or gum disease?

Teeth extracted because of pain, swelling, loose teeth, etc. have been removed because of gum disease or caries. Do not include teeth lost for other reasons, such as injury, orthodontics, or wisdom teeth removal.

$\begin{array}{lll}1 & \text { None } & \text { 8 Don't know/Not sure } \\ 2 & 1-5 & 9 \text { Refused } \\ 3 & 6 \text { or more, but not all } & \\ 4 & \text { All }\end{array}$

\section{3a. If you have teeth remaining:}

33a1. Do you presently have any restorations in your teeth? (white or silver fillings, gold or porcelain crowns or bridges)

1 Yes 8 Don't know/Not sure

2 No 9 Refused

33a2. Do you believe that you presently have any active decay/dental cavities in any of your teeth?

$\begin{array}{ll}1 \text { Yes } & 8 \text { Don't know/Not sure } \\ 2 \text { No } & 9 \text { Refused }\end{array}$

33a3. Do your gums often bleed when you brush or floss?

1 Yes 8 Don't know/Not sure

2 No $\quad 9$ Refused 
33a4. Are your gums often red, tender or swollen?

$\begin{array}{ll}1 \text { Yes } & 8 \text { Don't know/Not sure } \\ 2 \text { No } & 9 \text { Refused }\end{array}$

33a5. Do your gums pull away from your teeth in places?
1 Yes
8 Don't know/Not sure
2 No
9 Refused

33a6. Are your permanent teeth loose or separating?
1 Yes
8 Don't know/Not sure
2 No
9 Refused

33a7. Do you have any tartar/calculus or buildups on your teeth?
1 Yes
8 Don't know/Not sure
2 No
9 Refused

33a8. Do you frequently have bad breath?
1 Yes
8 Don't know/Not sure
2 No
9 Refused

33a9. Is the way your teeth bite together changing?

1 Yes 8 Don't know/Not sure

2 No $\quad 9$ Refused

34. If you have partial tooth loss, have you had all or some of your lost teeth replaced? This means you wear a denture, partial denture, bridge or dental implant.

1 Yes, I have replacements for all of my lost teeth.

2 Yes, I have replacements for some, but not all of my lost teeth.

3 No, I do not have replacements for any of my lost teeth. (SKIP TO

QUESTION \#43)

8 Don't know/Not sure

9 Refused

35. If you answered that you have replacements for some or all of your lost teeth, are your partials or bridges adequate?.Adequate means they are comfortable, they function and work well and they Look good Must meet all three criteria.
1 Yes
8 Don't know/Not sure
2 No
9 Refused

\section{PERSONS WITH DENTURES}

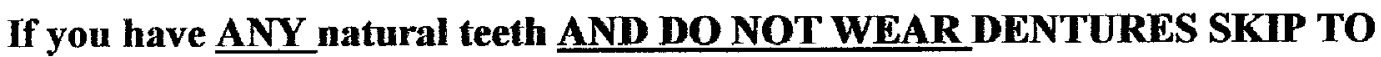
QUESTION \#43.

36. Do you have:

1 Upper and lower dentures

2 Upper denture only

3 Lower denture only

4 Implant supported denture

5 Other

8 Don't know/Not sure

9 Refused 
37. Which of these do you wear routinely?

1 Upper and lower dentures

5 Other

2 Upper denture only

8 Don't know/Not sure

3 Lower denture only

9 Refused

4 Implant supported denture

38. How often do you wear your dentures? Circle only one answer.

1 Every day

2 Some days

3 Only while eating

4 Never
5 Other

8 Don't know/Not sure

9 .Refused

39. How do you feel your dentures fit?
1 Excellent
3 Fair
8 Don't know/Not sure
2 Good
4 Poor
9 Refused

40. Do you have any sores or ulcers in your mouth or on your gums that you feel are caused by your dentures?

No Yes

If yes, how often do you have them?
1 Frequently
3 Rarely
8 Don't know/Not sure
2 Sometimes
9 Refused

\section{How long have you had your present dentures?}
1 Less than 1 year
$411-20$ years
Don't know/Not sure
$21-5$ years
$521-30$ years 9 Refused
3 6-10 years
$631+$ years

42. Do you feel you need new dentures made at this time?

No __ Yes

Don't know/Not sure

Refused

If Yes, you need new dentures made at this time, what is the reason? Circle all that apply.

1 Currently, don't have dentures

2 Current dentures are worn out

3 Teeth in dentures are broken or missing

4 Lost dentures

5 Not satisfied (appearance, way they feel)

6 Other

8 Don't know/Not sure

9 Refused 


\section{USE OF DENTAL HEALTH SERVICES}

43. How long has it been since you last visited a dentist or dental clinic for any reason?*

1 Within the past year ( 0 to 12 months ago)

2 Within the past two years ( 1 to 2 years ago)

3 Within the past five years ( 2 to 5 years ago)

45 or more years ago

5 Never

8 Don't know/Not sure

9 Refused

*Your best estimate

If you last went to the dentist under or EXACTLY I-year ago, mark "0-12 months ago." If you last went to the dentist from 1 year to EXACTLY 2 years ago, mark "1-2 years ago."

If you last went to the dentist from two years to five years ago, mark " 2 to 5 years ago." If you last went to the dentist EXACTLY or greater than 5 years ago, please mark "5 or more years ago."

If you are unsure, mark "Don't know/Not sure."

\section{If you have not visited a dentist in more than 1 year, what is the main} reason?

Circle only one answer (the MAIN reason)

1 Does not apply/have been to a dentist in the past year

2 Fear, apprehension, nervousness

3 Dislike going

4 Cost

5 Do not have/know a dentist

6 Cannot get to the office/clinic (too far away, no appointments available, no transportation)

7 No reason to go (no problems, no teeth)

8 Other priorities

9 Have not thought of it

10 Other

88 Don't know/Not sure

99 Refused 
45. What was the purpose of your most recent visit to the dentist? Circle only one answer (the MAIN reason)

1 Prevention/Cleaning

2 Pain/Dental Emergency/Extraction

3 Restorative/Fillings

4 Crowns/Bridges

5 Dentures/Partial Dentures

6 Gum Therapy/Periodontal

7 Root Canals/Endodontic Therapy

8 Other

\section{HEALTH ACCESS BELIEFS AND QUESTIONS}

46. Do you have any major barriers in getting dental care or services?

Yes No (SKIP TO QUESTION \#48)

If Yes, circle all that apply.

1 Do not have dental insurance

2 Don't like/trust/believe in dentists

3 Did not know where to go

4 Could not afford (no money)

5 Dentist did not accept Medicaid

6 Difficulty in getting appointment

7 No dentist available

8 No way to get there (transportation)

9 Limitation of mobility

10 Other

88 Don't know/Not sure

99 Refused

47. Which of the following services do you have difficulty in obtaining? Circle all that apply.

1 Basic dental services: check-ups, cleaning and fillings.

2 Advanced dental services: crowns, bridges, implants, periodontal treatment. and extractions.

3 Emergency dental services: able to make appointment and visit dentist right away for dental pain or oral problem.

4 Prosthodontic dental services: having dentures/partials made by dentist.

5 Other service (please list)

8 Don't know/Not sure

9 Refused 
48. What are your recommendations for improving your oral health status?

Circle all that apply or write in comment.

1 Make dentistry affordable.

2 Mobile clinic (mobile van comes to a specific location such as a senior center or church in your community)

3 House calls (Dentist or dental hygienist comes to your house)

4 Dental offices made more accessible for wheelchairs, parking, etc.

5 Other

8 Don't know/Not sure

9 Refused

THANK YOU FOR TAKING TIME TO RESPOND TO THIS QUESTIONNAIRE! 


\title{
APPENDIX B
}

\section{PARTICIPATING NURSING HOMES/ASSISTED LIVING FACILITIES}

\author{
Baptist Convalescent Center \\ 120 Main Street \\ Newport, KY 41071 \\ Baptist Towers \\ Carl S. Hennigen, Director \\ 800 Highland Avenue \\ Coventington, KY 41011 \\ Britthaven of Benton \\ Jennifer Thomas, Administrator \\ US Highway 641 South \\ P.O. Box 385 \\ Benton, KY 42025 \\ Britthaven of South Louisville \\ Robert Flatt, Administrator \\ 9600 Lamborne Boulevard \\ Louisville, KY 40272 \\ Brookfield Manor \\ Rebecca Hammonds, Administrator \\ 2820 Richard Street \\ P.O. Box 711 \\ Hopkinsville, KY 42240 \\ Carmel Home \\ Sr. Frances Teresa, Director \\ Camel Manor Road \\ Ft. Thomas, KY 41042 \\ Carmel Manor \\ Sr. Teresa Kennedy, Administrator \\ Carmel Manor Rd. \\ Ft. Thomas, KY 41075
}


Colonial Gardens

Ken Kaser, Manager

6900 Hopeful Road

Florence, KY 41042

Episcopal Church Home

Keith R. Knapp, Chief Executive Officer

1201 Lyndon Lane

Louisville, KY 40222-4398

Fern Terrace Lodge of Bowling Green

1030 Shive Lane

Bowling Green, KY 42103

Fern Terrace Lodge of Mayfield

A. Loudean Austin, Administrator

P.O. Box 325

Mayfield, KY 42066

Fern Terrace Lodge of Owensboro

45 Woodford Avenue

Owensboro, KY 42301

Four Courts Senior Center

Deborah A. May, Executive Director

2100 Millvale Road

Louisville, KY 40205

Franciscan Health Care Center

Suzanne Rinne, Administrator

3625 Fern Valley Road

Louisville, KY 40219

Good Shepherd Community Nursing Center

Priscilla Hager, Executive Director

P.O. Box 424

Phelps, KY 41553

Laurel Heights Home for the Elderly

Kathy Young, Administrator

208 West Twelfth Street

P.O. Box 1800

London, KY 40743-1800 
Laurel Village (Assisted Living)

Kathy Young, Administrator

P.O. Box 1920, 815 Mills St.

London, KY 40743-1920

Lexington Country Place

Katherine E. Davis, Administrator

700 Mason Headley Road

Lexington, KY 40504

Loretto Motherhouse Infirmary

Sr. Kay Carlew, Administrator

515 Nerinx Road

Nerinx, KY 40049

Metzmeier Nursing Home, Inc.

Don Metzmeier, Administrator

700 North Central Avenue

Campbellsville, KY 42718-2098

Paragon at West Park

Angela Butts, Community Director

4960 Village Square Drive

Paducah, KY 42301

Richmond Place (Assisted Living)

Carol A. Brinegar, Care Manager

3051 Rio Dosa Drive

Lexington, KY 40509

Rockcastle Health and Rehabilitation

7190 Main St.

Brodhead, KY 40409

Sacred Heart Village

Leslie Wilson, President/CEO

2120 Payne Street

Louisville, KY 40206

Stanton Nursing \& Rehabilitation Center

31 Derickson Lane

Stanton, KY 40380 
Woodland Oaks

Paula F. Long Barker, Administrator

1820 Oakview Road

P.O. Box 1309

Ashland, KY 41105-1309

Pine Meadows Health Care, Inc.

Jamie Gitzinger, Administrator

1608 Hill Rise Drive

Lexington, KY 40504 


\section{CURRICULUM VTTAE}

NAME

BUSTNESS ADDRESS

1981

1978
Lynn Donnelly Austin

Western Kentucky University

Department of Allied Health

Program of Dental Hygiene

1906 College Heights Blvd. \#11032

Bowling Green, Kentucky 42101

Western Kentucky University

University of Louisville

Joint Doctoral Program (Ph.D.)

Higher Education Administration

University of Michigan

Master of Public Health

School of Public Health

Dental Public Health

Ann Arbor, Michigan

University of Michigan

Bachelor of Science

School of Dentistry

Dental Hygiene Program

Ann Arbor, Michigan

\section{CREDENTIALS}

National Dental Hygiene Board Examination (1978)

Registered Dental Hygienist (R.D.H.)

Licensure:

Kentucky 1982 - present

Michigan 1978 - 1992 


\section{PROFESSIONAL EXPERIENCE}

January 2006 Department Head, Department of Allied Health Program Director, Program of Dental Hygiene Western Kentucky University, Bowling Green, Kentucky

January 2005 Interim Department Head, Department of Allied Health Program Director, Program of Dental Hygiene Western Kentucky University, Bowling Green, Kentucky

1988-2005 Associate Professor, Program of Dental Hygiene, Department of Allied Health, Western Kentucky University, Bowling Green, Kentucky

1985-1988 Assistant Professor, Program of Dental Hygiene, Department of Allied Health, Western Kentucky University, Bowling Green, Kentucky

1982-1985 Instructor, Program of Dental Hygiene, Department of Allied Health, Western Kentucky University, Bowling Green, Kentucky

\section{LEADERSHIP APPOINTMENTS}

American Dental Hygienists' Association (ADHA)

- Advisor to Student Members of the American Dental Hygienists' Association (SADHA) (1984-present)

American Dental Education Association (ADEA)

- Liaison to the AADS Geriatric Project (1989)

- Community Dental Health Competency Revision Committee (2005)

American Dental Association

- Alternate, Dental Hygiene Test Construction Committee (1996-1999)

- Member, Dental Hygiene Test Construction Committee Component A-III (2000-2006)

- Member, Dental Hygiene Test Construction Committee Component B (20062011)

- Member, Joint Commission on National Dental Boards (2005-2012)

- Member, Site Visitor Team, Joint Commission on Dental Accreditation (2001-2007) 
Sigma Phi Alpha

- Secretary-Treasurer, Chi Alpha Chapter, Western Kentucky University, (19951997)

- President, Chi Alpha Chapter, Western Kentucky University, (1997-present)

Kentucky Dental Hygienists' Association (KDHA)

- Vice-President (1997-1998)

- Annual Session Program Chair (1997-1998)

- Session Table Clinic Chair (1996-1997)

- Vice-President (1994-1995)

- Annual Session Program Chair (1994-1995)

- Speaker of the House of Delegates (1989-1992)

- President (1987-1988)

- Vice-President (1986-1987)

South Central Kentucky Dental Hygienists' Society (SCKDHS)

- Trustee (1985-1986)

- Secretary (1984-1985)

- National Children's Dental Health Month Chair (1983-1984)

- Kentucky Consortium on Dental Auxiliary Education (KCDAE) Secretary/Treasurer (1985-1986)

\section{RESEARCH AND CREATIVE ACTIVITIES \\ Publications (since 1998)}

"Addressing Health Disparities: A Model for Improving Status of Dental Health in the World," (abstract) Association of Schools of Allied Health Professions Annual Conference, 2005.

"Treating the Underserved Dental Patient: An Approach to Addressing Disparities in Dental Health," (abstract) Association of Schools of Allied Health Professions Annual Conference, 2005.

"Sealing Our Community: A Model for a Preventive Dental Public Health Program," (abstract) Association of Schools of Allied Health Professions Annual Conference, 2004.

Austin, LD, "Implant Dentistry: An Option for Patients of Any Age," Access, March 2003.

Austin, LD, "Mock Board Exams as Predictors on the National Dental Hygiene Board Exam," accepted for presentation at the American Dental Education Association Conference, March 2003, San Antonio, TX, (with publication in ADEA journal).

Austin, LD, "Clinical and Classroom Application of Digital Photography and Radiography," Access, May-June 2002. 
Austin, LD, "A Model for Community Programs: Addressing Access to Care," Journal of Dental Hygiene, November 2001.

Austin, LD, "The Use of Case Studies to Teach in Science," Exploring the Scholarship of Teaching, Center for Teaching and Learning, February 1, 2001.

Austin, LD, Crafton, B, "The Bulimic Patient: Confrontation, Intervention, Referral," Access, December 2000.

Crafton, B., Austin, LD., "Periodontal Management of Refractory, Rapidly Progressive Periodontitis," Access, Nov. 1999.

Austin, LD, Crafton, B., "Periodontal Management of Adult Periodontitis Exacerbated by Risk Factors," Access, Sept.-Oct. 1999.

Austin, LD, "A Model for Interdisciplinary Collaboration," National Area Health Education Consortium Proceedings, Louisville, Kentucky, Fall 1999.

Austin, LD, "Pins and Needles: A Case Study in the Management of Occupational Exposure to Percutaneous Injuries,"

http://ublib.buffalo.edu/libraries/projects5/cases/ubcase.htm, September 1998.

\section{Presentations (since 1990)}

"Addressing Health Disparities: A Model for Improving Status of Dental Health in the World," Association of Schools of Allied Health Professions Annual Conference, Houston, TX, Oct. 2005.

"Treating the Underserved Dental Patient: An Approach to Addressing Disparities in Dental Health," Association of Schools of Allied Health Professions Annual Conference, Houston, TX, Oct. 2005.

Sealing Our Community: A Model for a Preventive Dental Public Health, Association of Schools of Allied Health Professions Annual Conference, Tampa, FL, Oct. 2004.

"Mock Board Exams as Predictors on the National Dental Hygiene Board Exam," American Dental Education Association Annual Conference in San Antonio, TX, March 2003.

"A Model for Community Programs: Addressing Access to Care," American Dental Hygienists' Association Annual Meeting, Nashville, TN, June 2001.

"Infection Control in the Dental Environment," June 4, 1992, American Dental Hygienists' Association, Louisville, Kentucky. 
"Cleft Lip and Cleft Palate: The Team Approach," September 22, 1990, Twelfth Annual Interdisciplinary Health Care Team Conference, Indianapolis, Indiana. 